\title{
Increased apoptosis and browning of TAK1-deficient adipocytes protects against obesity
}

\author{
Antonia Sassmann-Schweda, ${ }^{1}$ Pratibha Singh, ${ }^{1}$ Cong Tang, ${ }^{1}$ Astrid Wietelmann, ${ }^{2}$ \\ Nina Wettschureck, ${ }^{1,3}$ and Stefan Offermanns $\mathbf{s}^{1,3}$ \\ 'Department of Pharmacology and 'Scientific Service Group Nuclear Magnetic Resonance Imaging, Max Planck Institute \\ for Heart and Lung Research, Bad Nauheim, Germany. ${ }^{3}$ Medical Faculty, Goethe-University Frankfurt, Frankfurt, Germany.
}

Obesity is an increasing health problem worldwide, and nonsurgical strategies to treat obesity have remained rather inefficient. We here show that acute loss of TCF- $\beta$-activated kinase 1 (TAK1) in adipocytes results in an increased rate of apoptotic adipocyte death and increased numbers of M2 macrophages in white adipose tissue. Mice with adipocyte-specific TAK1 deficiency have reduced adipocyte numbers and are resistant to obesity induced by a high-fat diet or leptin deficiency. In addition, adipocyte-specific TAK1-deficient mice under a high-fat diet showed increased energy expenditure, which was accompanied by enhanced expression of the uncoupling protein UCP1. Interestingly, acute induction of adipocyte-specific TAK1 deficiency in mice already under a high-fat diet was able to stop further weight gain and improved glucose tolerance. Thus, loss of TAK1 in adipocytes reduces the total number of adipocytes, increases browning of white adipose tissue, and may be an attractive strategy to treat obesity, obesity-dependent diabetes, and other associated complications.

\section{Introduction}

An imbalance of dietary energy uptake and energy consumption is one of the predominant health problems in developed countries, and an increasing proportion of the population has a positive energy balance, resulting in obesity with various health consequences, such as increased risk for type 2 diabetes, cardiovascular diseases, and cancer (1-3). Recent work has shown that obesity induces a chronic low-grade inflammation in adipose tissue that is accompanied by the infiltration of adipose tissue by macrophages and increased levels of cytokines, such as IL- 6 and TNF- $\alpha$ (4-7). Current models suggest that overnutrition induces cellular, metabolic, and inflammatory stress in adipocytes, resulting in the release of chemokines and other factors that attract macrophages. Adipose tissue macrophages then release chemokines and cytokines, which further promote adipose tissue inflammation (8). Studies in mice and in isolated adipocytes have shown that inflammatory signaling pathways involving TLR4, c-Jun N-terminal kinase 1 (JNK1), or nuclear factor- $\mathrm{B}(\mathrm{NF}-\kappa \mathrm{B})$ play an important role in obesity-associated adipose tissue inflammation and the development of insulin resistance (9-14).

It had been the prevailing view that the number of adipocytes in the fat tissue of adult organisms is constant, and that an increase in fat mass during the development of obesity primarily results from changes in adipocyte volume (15). However, recent data provided evidence that adipose tissue undergoes a constant turnover, with preadipocytes differentiating into mature adipocytes and mature adipocytes dying at the

Conflict of interest: The authors have declared that no conflict of interest exists.

Submitted: March 3, 2016

Accepted: April 12, 2016

Published: May 19, 2016

Reference information: JCI Insight. 2016;1(7):e81175. doi:10.1172/jci.insight.81175. same rate, resulting in an annual renewal of approximately $10 \%$ of human adipocytes (16). While adipocyte hypertrophy can be seen in all forms of obesity, an increase in adipocyte number is more likely to be seen in severely obese patients (17). However, the fat cell number does not decrease in adult humans, even after a substantial weight loss $(16,18,19)$.

In searching for potential targets that can be exploited to modify both proinflammatory signaling in adipocytes and adipocyte turnover, we focused on TGF- $\beta$-activated kinase 1 (TAK1), which couples TLRs and various cytokine receptors to the activation of the $\mathrm{NF}-\kappa \mathrm{B}$ pathway as well as to mitogen-activated kinases, such as p38 and JNK $(20,21)$. Thereby, TAK1 plays a well-established role in proinflammatory signaling. In addition, inhibition of TAK1 function has been shown to promote programmed cell death in 
vitro and in vivo, an effect believed to result from the loss of TAK1-mediated survival signaling (22-28). Here we show that induced loss of TAK1 in mature adipocytes increases adipocyte apoptosis and turnover and leads to elevated white adipose tissue (WAT) thermogenesis, resulting in reduced high-fat diet-induced obesity and insulin resistance.

\section{Results}

TAK1 deficiency in adipocytes leads to increased rate of apoptosis. To generate inducible white adipocyte-specific TAK1-deficient mice, we crossed the AdipoqCreER ${ }^{\mathrm{T} 2}$ mouse line (29) with mice carrying a floxed allele of TAK1 (30). Adipocyte-specific, tamoxifen-inducible TAK1-deficient mice (AdipoqCreER ${ }^{\mathrm{T} 2+/-; \mathrm{TAK}}{ }^{\mathrm{f} / / \mathrm{fl}}$, henceforth termed $A d \mathrm{TAK} 1-\mathrm{KO}$ ) were viable and fertile. Isolated adipocytes of tamoxifen-induced $A d \mathrm{TAK} 1-\mathrm{KO}$ mice showed strong recombination of floxed Tak1 alleles in white but not brown adipocytes, resulting in loss of TAK1 protein (Figure 1A). To analyze the cellular consequence of acutely induced TAK1 deficiency in adipocytes, we tested the effect of TNF- $\alpha$ on effectors downstream of TAK1. In WAT explants of control animals, TNF- $\alpha$ induced time-dependent phosphorylation of JNK, whereas this effect was strongly attenuated in WAT explants of AdTAK1-KO mice (Figure 1B). Also, TNF- $\alpha$-induced activation of the NF- $\mathrm{kB}$ pathway was abrogated in WAT explants from AdTAK1-KO mice compared with control, as indicated by the loss of TNF- $\alpha$-induced phosphorylation and degradation of $\mathrm{I} \kappa \mathrm{B} \alpha$ (Figure $1 \mathrm{C}$ ).

To test whether TAK1-deficient adipocytes are prone to undergo programmed death in vitro, we isolated adipocytes from AdTAK1-KO and control (wild-type) mice and cultured them for 72 hours. Whereas freshly isolated wild-type and TAK1-deficient adipocytes did not show any obvious morphological differences, AdTAK1-KO adipocytes, but not control adipocytes, underwent massive cell death within 2-3 days after isolation (Figure 1D). The death of TAK1-deficient adipocytes was accompanied by increased caspase-3 activity and was blocked by the caspase inhibitor Z-VAD-FMK (Figure 1D), indicating increased apoptosis in vitro. Also, in vitro induction of isolated adipocytes from Adipoq-CreER ${ }^{\mathrm{T} 2}$;AK $1^{\mathrm{f} / \mathrm{fl}}$ mice with 4-OH-tamoxifen resulted in increased apoptosis (Figure 1E), indicating that it is an adipocyte-autonomous phenomenon. TUNEL assays in adipose tissue sections from wild-type and AdTAK1-KO mice revealed more TUNEL-positive cells in the adipose tissue of AdTAK1-KO mice (Figure 1F). We then determined caspase-3 activity in freshly isolated adipocytes from wild-type and AdTAK1-KO mice fed with chow or a high-fat diet (HFD) or on a leptin-deficient background $(o b / o b)$. Caspase-3 activity was increased in TAK1-deficient adipocytes under all conditions (Figure 1G), indicating increased apoptosis of adipocytes also under in vivo conditions. In some systems it has been shown that TAK1 deficiency sensitizes cells to TNF- $\alpha$-induced apoptosis $(24-26,31)$. However, we did not observe an increased apoptosis of TAK1deficient adipocytes in response to TNF- $\alpha$ treatment, and fat cell apoptosis was not affected by the TNF- $\alpha$ blocker etanercept in vitro or in vivo (Figure $1 \mathrm{H}$ ).

Reduced adipocyte number and increased number of M2 macrophages in AdTAK1-KO mice. Next, we investigated the adipose tissue morphology of $A d \mathrm{TAK} 1-\mathrm{KO}$ and control animals either on chow or an HFD or on an $o b / o b$ background 16 weeks after tamoxifen induction. Histological analysis revealed no differences between AdTAK1-KO and control mice with regard to the volume of adipocytes from epididymal and subcutaneous adipose tissue (Figure 2, A-C). However, the total number of adipocytes in epididymal and inguinal subcutaneous fat tissue was significantly reduced in $A d \mathrm{TAK} 1-\mathrm{KO}$ mice under normal chow as well as under an HFD and $o b / o b$ background (Figure 2, D and E). Pref-1-positive preadipocytes in the stromal vascular fraction (SVF) of AdTAK1-KO mice showed increased BrdU incorporation (Figure 2F), indicating partial compensation of increased adipocyte death by enhanced proliferation.

Low-grade chronic inflammation of adipose tissue characterized by macrophage invasion is a hallmark of obesity and insulin resistance $(8,11)$. To investigate how loss of TAK1-mediated signaling in adipocytes affected adipose tissue inflammation, we studied macrophage infiltration in control and AdTAK1-KO mice. We found an increased expression of macrophage marker genes such as Cd68, Emr1, and Cd11b under both chow and HFD (Figure 3A), and flow cytometric analyses showed elevated numbers of macrophages in the SVF of chow-fed and HFD-fed AdTAK1-KO mice (Figure 3B). This was accompanied by a significantly increased number of crown-like structures (Figure 3C), indicating macrophages surrounding dying or dead adipocytes (32). Gene expression analysis revealed increased expression of M2 macrophage marker genes in adipose tissue of $A d \mathrm{TAK} 1-\mathrm{KO}$ mice, whereas expression of M1 marker genes was unchanged or even decreased (Figure 3D). Flow cytometry using the SVF confirmed a larger number of M2 macrophages in adipose tissue of $A d \mathrm{TAK} 1-\mathrm{KO}$ mice (Figure 3E). The increase in M2-polarized macrophages 
A

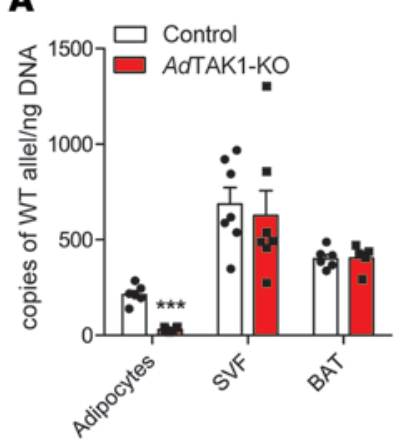

B

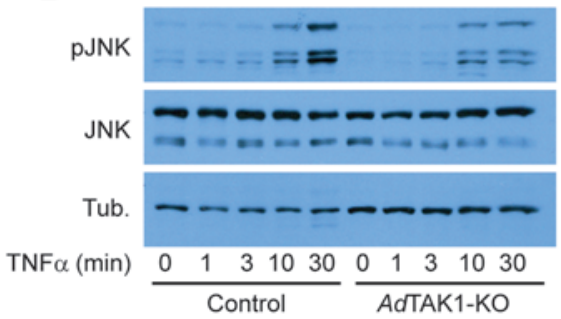

D
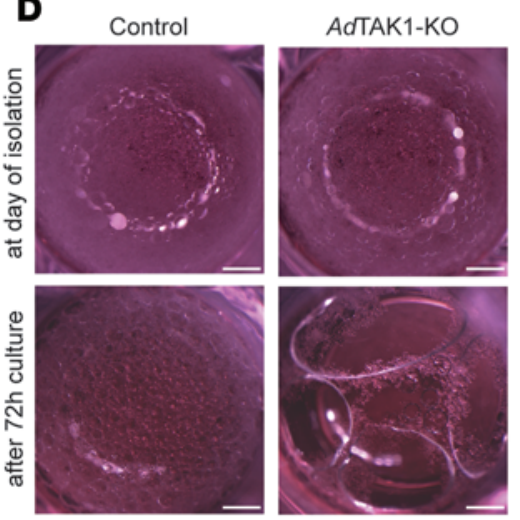

G

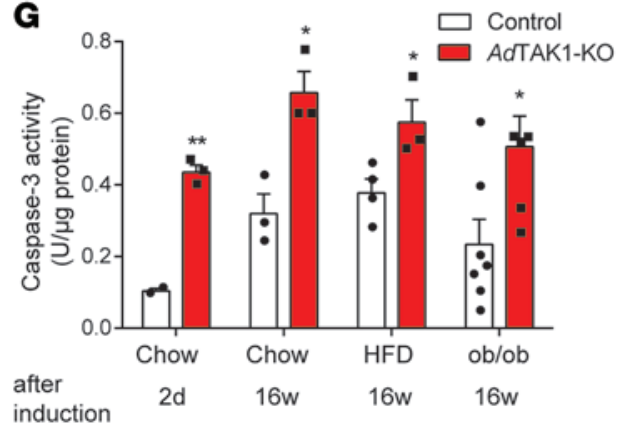

Adipocytes SVF BAT

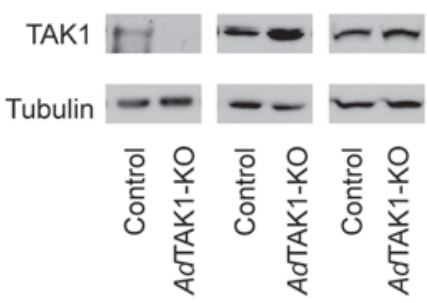

C
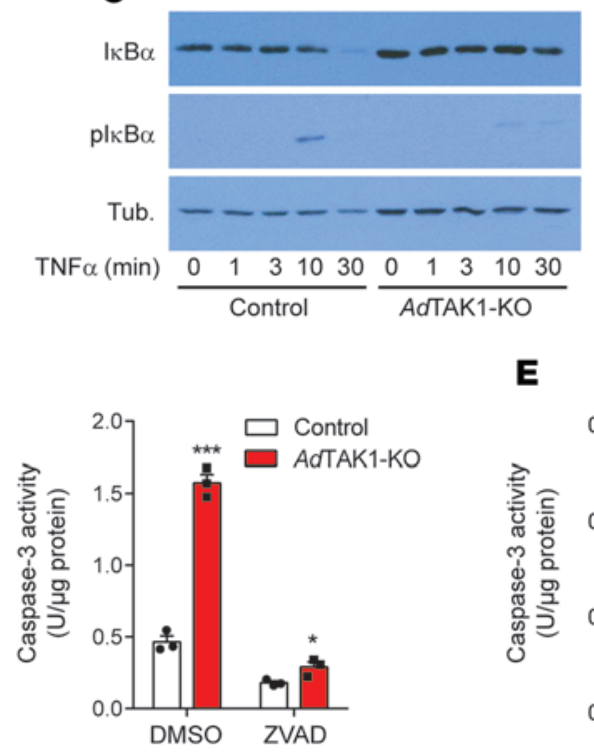

E

$\mathbf{F}$

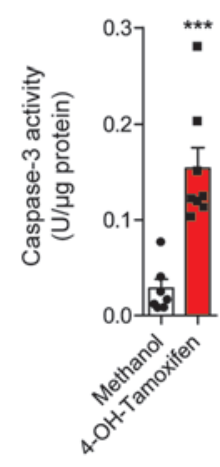

in vitro

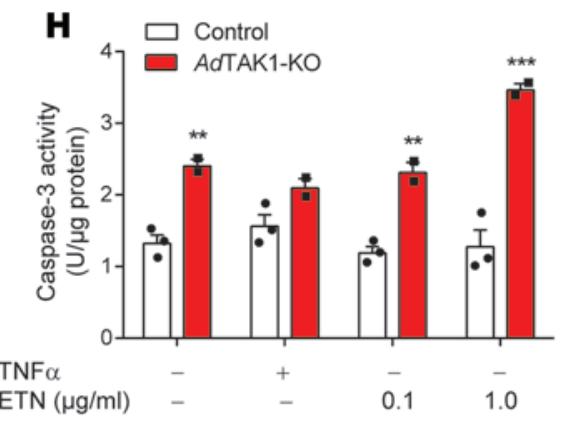

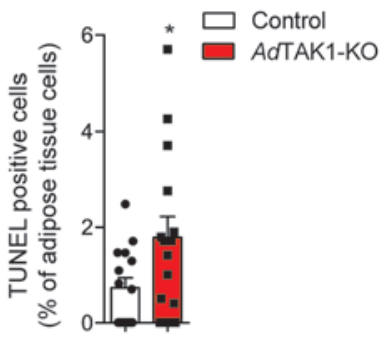

in vivo

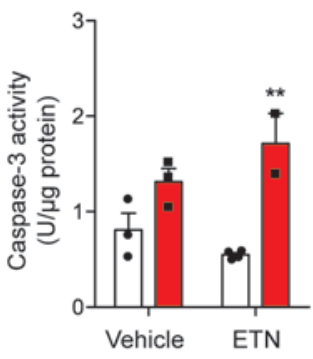

Figure 1. Consequences of TAK1 deficiency in white adipocytes. (A) Quantitative PCR (left) and Western blot (right) showing levels of TAK1 RNA or protein, respectively, in adipocytes and the stromal vascular fraction (SVF) of epididymal WAT and in BAT after tamoxifen induction ( $n=5-7 /$ group). (B and C) Effect of TNF- $\alpha$ on phosphorylation of JNK (B) as well as IKB $\alpha$ phosphorylation and degradation (C) in epididymal WAT explants from wildtype (control) and AdTAK1-KO mice. (D) Images of isolated epididymal adipocytes from AdTAK1-KO and wild-type (control) mice at day of isolation and after 72 hours of ex vivo culture (left panels). Right panel: statistical evaluation of caspase-3 activity in the absence or presence of $20 \mu M$ Z-VAD-FMK (ZVAD) after 48 hours of adipocyte culture $(n=3 /$ group). Scale bars: $1 \mathrm{~mm}$. (E) Effect of $4-\mathrm{OH}$-tamoxifen treatment of isolated epididymal adipocytes from Adipoq-CreER ${ }^{\mathrm{T2}} ; \mathrm{TAK}^{\text {fl/fi }}$ mice on caspase activity ( $n=7-8 /$ group). (F) Analysis of TUNEL staining of epididymal adipose tissue from wild-type (control) and AdTAK1-KO mice ( $n=15$ /group). (G) Caspase-3 activity in freshly isolated epididymal adipocytes from AdTAK1-KO and wild-type (control) mice 2 days after tamoxifen induction, 16 weeks after tamoxifen induction, 16 weeks after tamoxifen induction and HFD feeding, and 16 weeks after tamoxifen induction in $o b / o b$ background animals ( $n=3-7 /$ group). (H) Effect of TNF- $\alpha$ and etanercept (ETN) on caspase-3 activity in epididymal adipocytes prepared from wild-type (control) and AdTAK1-KO mice ( $n=3 /$ group). Right panel: AdTAK1-KO and control mice were treated with etanercept $(100 \mathrm{mg} / \mathrm{kg})$ in vivo on days 1,3 , and 5 after tamoxifen induction, and caspase-3 activity was determined in freshly isolated adipocytes from these animals $(n=3-4)$. Shown are mean values $\pm \mathrm{SEM} ;{ }^{*} P \leq 0.05,{ }^{* *} P \leq 0.01,{ }^{* *} P \leq 0.001$ (ANOVA [D, G, and $\left.\mathbf{H}\right]$ or $t$ test $[\mathbf{A}, \mathbf{E}$, and $\mathbf{F}]$ ). 
A

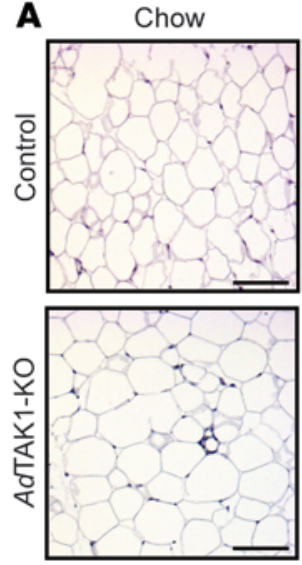

HFD
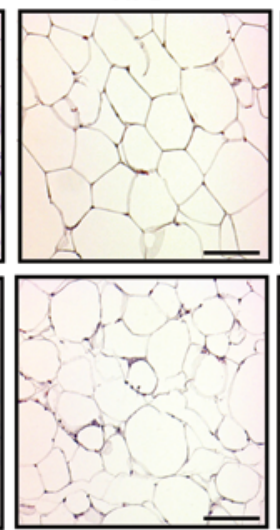

ob/ob

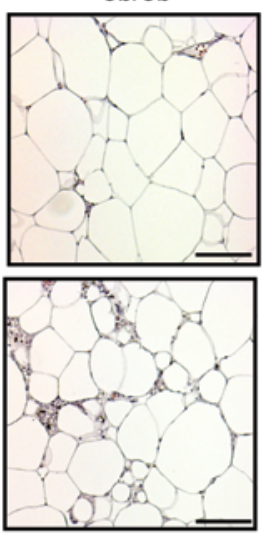

Figure 2. White adipose tissue composition in the absence of TAK1. (A) H\&E-stained epididymal adipose tissue from AdTAK1-KO and wild-type (control) mice fed chow or an HFD for 16 weeks or on an $o b / o b$ background. Scale bars: $100 \mu \mathrm{m}$. (B-E) Average adipocyte volume (B and $\mathbf{C}$ ) and adipocyte number ( $\mathbf{D}$ and $\mathbf{E}$ ) in epididymal (B and $\mathbf{D}$ ) and subcutaneous inguinal ( $\mathbf{C}$ and $\mathbf{E}$ ) adipose tissue of AdTAK1-KO and wild-type (control) mice fed chow or an HFD for 16 weeks or on an ob/ob background ( $n=4$ /group). (F) Flow cytometric analysis of proliferating pre-adipocytes in wild-type (control) and AdTAK1-KO mice fed an HFD for 16 weeks ( $n=4-5 /$ group). Shown are mean values \pm SEM. ${ }^{*} P \leq 0.05,{ }^{* *} P \leq 0.001$ (ANOVA [B-E] or $t$ test [F]).

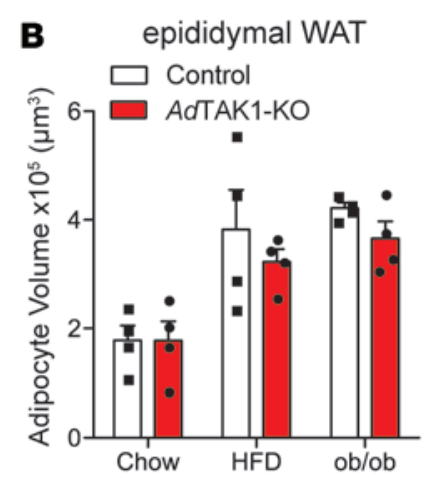

D epididymal WAT
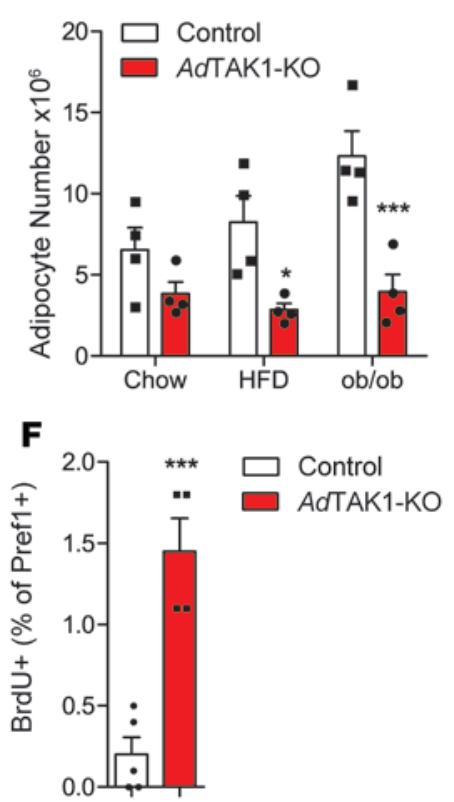

C inguinal subcutaneous WAT

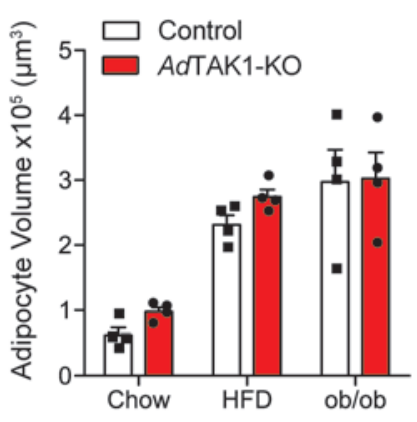

E inguinal subcutaneous WAT

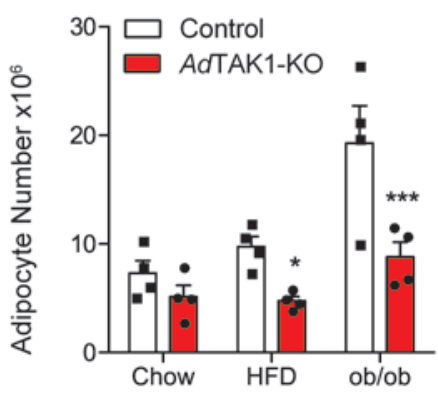

was accompanied by increased tyrosine hydroxylase expression (Figure 3F).

Development of body weight and adipose tissue mass in AdTAK1-KO mice. To study the long-term consequences of increased adipocyte apoptosis in AdTAK1-KO mice, we determined body weight gain over 16 weeks on a chow diet or an HFD or on an $o b / o b$ background. Whereas chow-fed mice did not show clear differences in body weight (Figure 4A), weight gain in HFD-fed $A d$ TAK1-KO mice and $A d \mathrm{TAK} 1-\mathrm{KO}-\mathrm{ob} / \mathrm{ob}$ mice was clearly reduced (Figure 4, B and C). This effect was due to reduced WAT mass, whereas body length and the weight of other organs, such as the liver, were not changed (Figure 4D and data not shown). The weight of epididymal and subcutaneous adipose tissue was reduced in AdTAK1-KO mice kept on normal chow or an HFD or when on an $o b / o b$ background (Figure 4E), whereas the weight of brown adipose tissue (BAT) was unchanged (Figure $4 \mathrm{~F}$ ). The overall reduction of adipose tissue mass of AdTAK1-KO mice compared to control animals under chow-fed, HFD-fed, and $o b / o b$ background conditions was also obvious from magnetic resonance imaging (Figure 4G).

Increased thermogenesis and glucose tolerance in AdTAK1-KO mice. Interestingly, food consumption by $A d \mathrm{TAK} 1-\mathrm{KO}$ mice was elevated compared to control animals under HFD-fed and $o b / o b$ background conditions (Figure 5A), while no differences in activity (Figure $5 \mathrm{~B}$ ) or in respiratory quotient (Figure 5C) were observed. In line with increased food intake and decreased body weight, whole-body

energy expenditure and energy expenditure adjusted for body weight were increased in AdTAK1-KO mice fed an HFD or on an $o b / o b$ background (Figure 5, D-G). This led us to explore whether the increased whole-body energy turnover was associated with "browning" in WAT. Indeed, UCP-1 protein levels (data not shown) and mRNA levels of UCP-1 and other markers for beige adipocytes were increased severalfold in adipocytes from WAT of mice on an HFD or an $o b / o b$ background (Figure 5H).

We then tested whether decreased body fat mass in AdTAK1-KO mice had an effect on glucose 
A

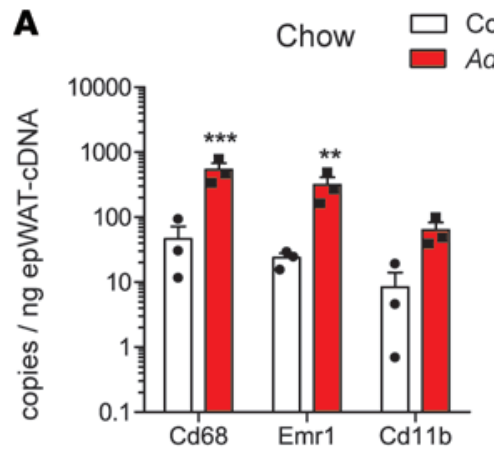

C

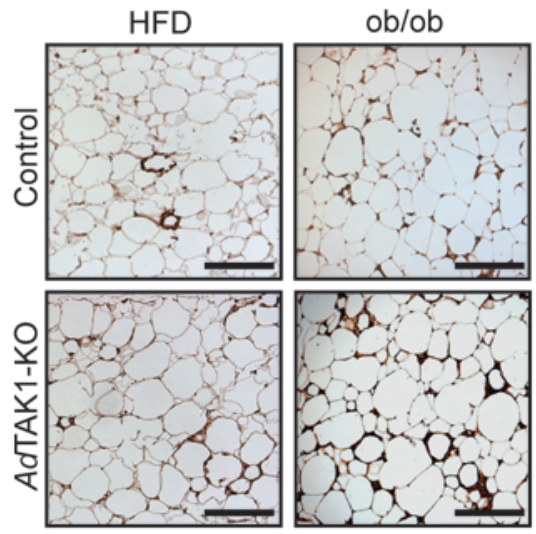

HFD

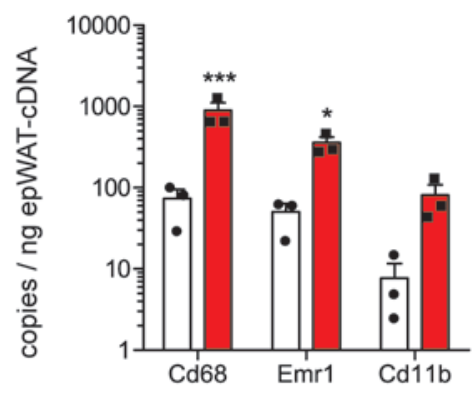

B $\square$ Control

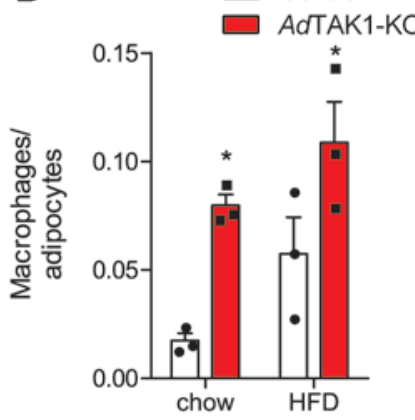

D

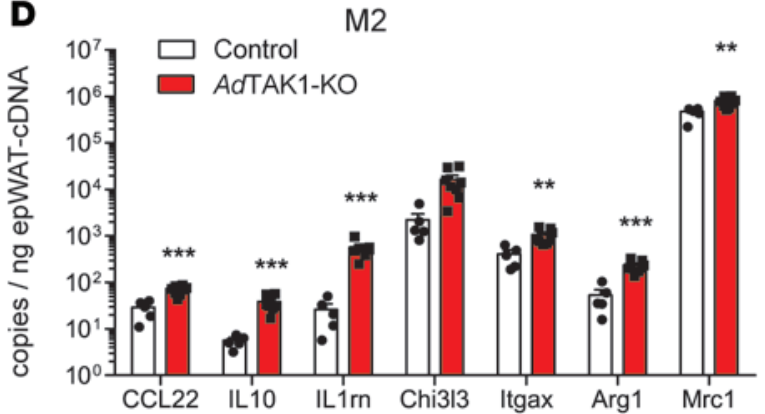

epididymal WAT

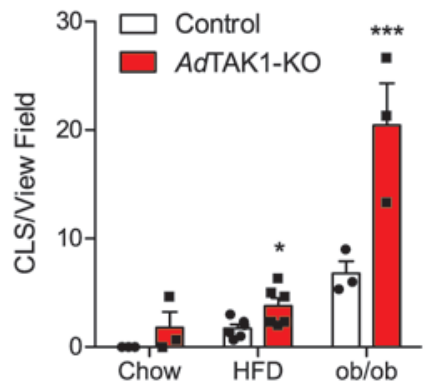

inguinal subcutaneous WAT

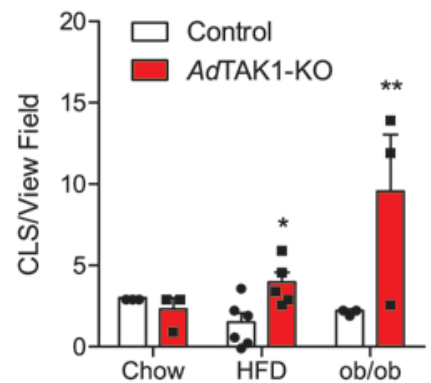

E

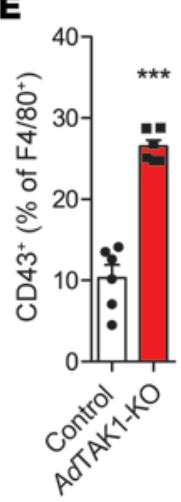

$\mathbf{F}$

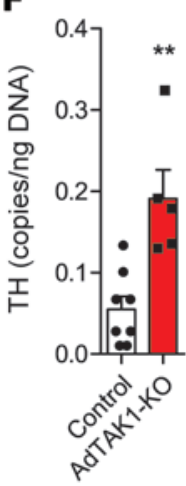

Figure 3. Analysis of adipose tissue inflammation in AdTAK1-KO mice. (A) Expression of macrophage marker genes in epididymal white adipose tissue from AdTAK1-KO and wild-type (control) mice fed chow or an HFD ( $n=3 /$ group). (B) Macrophage content analyzed by flow cytometry of the stromal vascular fraction from AdipoqCreER ${ }^{\mathrm{T2}} ; \mathrm{TAK}^{\mathrm{fl} / \mathrm{fl}}$ and wild-type (control) mice fed chow or an HFD ( $n=3 /$ group). (C) Mac2-stained epididymal adipose tissue and number of crown-like structures in epididymal and inguinal subcutaneous adipose tissue of wild-type (control) and AdTAK1-KO mice fed an HFD or kept on an ob/ob background ( $n=3-6 /$ group). Scale bars: 200 $\mu \mathrm{m}$. (D) Expression of M1 and M2 macrophage marker genes in epididymal adipose tissue of AdTAK1-KO and wild-type (control) mice after 16 weeks of an HFD ( $n=5-8 /$ group). (E) Percentage of CD43-positive macrophages in the stromal vascular fraction isolated from epididymal adipose tissue from AdTAK1-KO and wild-type (control) mice ( $n=6 /$ group). (F) Tyrosine hydroxylase (TH) expression by adipocytes from epididymal WAT of wild-type (control) and AdTAK1-KO mice fed an HFD ( $n=5-8 /$ group). Shown are mean values \pm SEM. ${ }^{*} P \leq 0.05,{ }^{* *} P \leq 0.01,{ }^{* *} P \leq 0.001$ (ANOVA [A-D] or $t$ test [E and $\mathbf{F}]$ ).

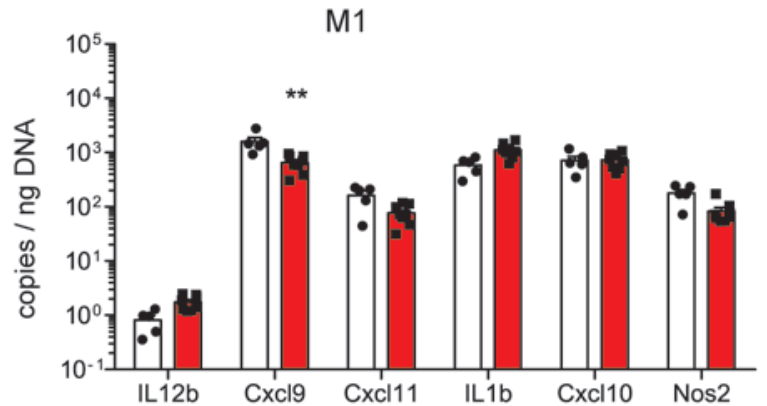


metabolism. Under chow-fed conditions we could not detect any differences in glucose tolerance between $A d \mathrm{TAK} 1-\mathrm{KO}$ and control mice, but $A d \mathrm{TAK} 1-\mathrm{KO}$ mice showed improved glucose tolerance after HFD feeding and on an $o b / o b$ background when compared to wild-type animals (Figure 6A). In euglycemichyperinsulinemic clamp studies, we observed no differences in glucose infusion rate (GIR) between chowfed wild-type and AdTAK1-KO animals, but GIR was increased compared to control animals in HFD-fed AdTAK1-KO mice, indicating improved insulin sensitivity (Figure 6B). Among various metabolic hormones, only plasma levels of insulin, leptin, and resistin were slightly reduced in chow- and HFD-fed AdTAK1-KO mice compared to wild-type animals (Figure 6C), further indicating an improved metabolic situation in AdTAK1-KO mice compared to control animals. In line with this, plasma levels of TNF- $\alpha$ and IL-6 were comparable or even decreased in AdTAK1-KO mice compared to wild-type animals under different conditions (Figure 6D). This improved metabolic situation is not an effect of alterations in lipolytic activity or glucose uptake per se, since basal and isoproterenol-stimulated lipolysis (Figure 6E) as well as basal and insulin-stimulated glucose uptake (Figure 6F) were unchanged in wild-type and AdTAK1-KO epididymal adipocytes, and expression levels of genes regulating lipid and glucose metabolism were similar in wild-type and $A d \mathrm{TAK} 1-\mathrm{KO}$ epididymal adipocytes (Figure 6, G and $\mathrm{H}$ ). In addition, liver triglyceride content was unchanged (Figure 6I).

Beneficial effects after induction of adipocyte-specific TAK1 deficiency in established obesity. Finally, we investigated whether loss of TAK1 in adipocytes also resulted in decreased weight gain and improved metabolic parameters when it was induced in mice with already established obesity. Tamoxifen induction was therefore started after feeding mice an HFD for 8 weeks, and HFD feeding was continued for another 16 weeks after induction of adipocyte-specific TAK1 deficiency. Body weight gain was indistinguishable between noninduced Adipoq $\mathrm{CreER}^{\mathrm{T} 2}$; $\mathrm{TAK}^{\mathrm{f} / \mathrm{fl}}$ and control animals during the first 8 weeks of HFD feeding (Figure 7A), and metabolic parameters such as intraperitoneal glucose tolerance (Figure 7C) and basal plasma insulin levels (Figure 7D) were unchanged. However, after tamoxifen induction, weight gain of AdTAK1-KO mice slowed down compared to control mice, and after 16 weeks of continuous HFD feeding, their body weight was significantly lower compared to control animals (Figure 7A), despite the fact that AdTAK1-KO mice ate slightly more than control animals (Figure 7B). In addition, glucose tolerance was improved and basal insulin levels did not further increase in AdTAK1-KO mice (Figure 7, C and D). Similarly, adipocyte number was decreased in epididymal adipose tissue of AdTAK1-KO mice, whereas adipocyte volume was unchanged (Figure 7E). These experiments show that, even in states of established obesity, inhibition of TAK1 can improve metabolic parameters and lower body weight.

\section{Discussion}

Severe human obesity is characterized by both an increase in fat cell volume as well as an increase in adipocyte number. The total number of adipocytes in lean and obese individuals appears to be established until the age of about twenty and stays constant during adulthood even after a substantial weight loss (16, $18,19,33)$. Recent evidence shows that adipocytes turn over at a rate of $10 \%$ annually and that the rate of cell turnover is the same in lean and obese individuals (16). Here we show that the induced loss of TAK1 selectively in adipocytes results in an increased rate of apoptotic adipocyte death, which is only partially compensated by increased adipocyte formation. This results in a reduced steady-state adipocyte number and a reduced fat mass, and AdTAK1-KO mice gained less weight under an HFD or when crossed onto an $o b / o b$ background. They also developed fewer secondary consequences of obesity, such as insulin resistance. Thus, an induced increase in the rate of adipocyte death can tip the balance of adipocyte birth and death, resulting in a reduction of total fat mass with beneficial metabolic consequences.

The development of obesity is accompanied by a chronic low-grade inflammation of adipose tissue, and evidence has accumulated that adipose tissue inflammation is an important factor for the development of obesity complications, including type 2 diabetes $(7,8,11,12)$. Parallel to the infiltration of adipose tissue by macrophages during obesity development, an increased rate of adipocyte death was observed $(32,34)$, and it has been suggested that chronic inflammation of obese fat tissue is a consequence of increased adipocyte death (32). However, whether a blockade of apoptotic cell death reduces fat tissue inflammation and the metabolic consequences of obesity is not clear. While an inhibition of apoptosis in the whole organism in global Bid-deficient mice reduced obesity-associated adipose tissue inflammation and its systemic metabolic complications (34), induction of massive adipocyte-specific apoptotic death induced infiltration of alternatively activated M2 macrophages but not of proinflammatory M1 macro- 
A

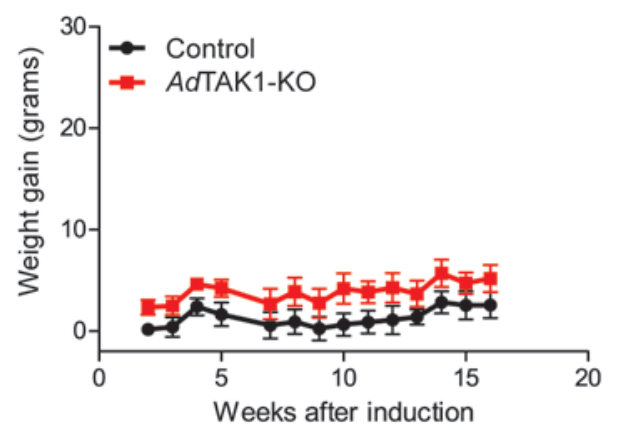

$\mathbf{B}$

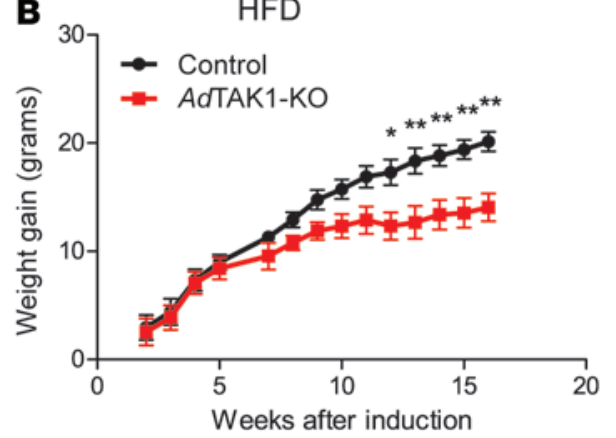

C
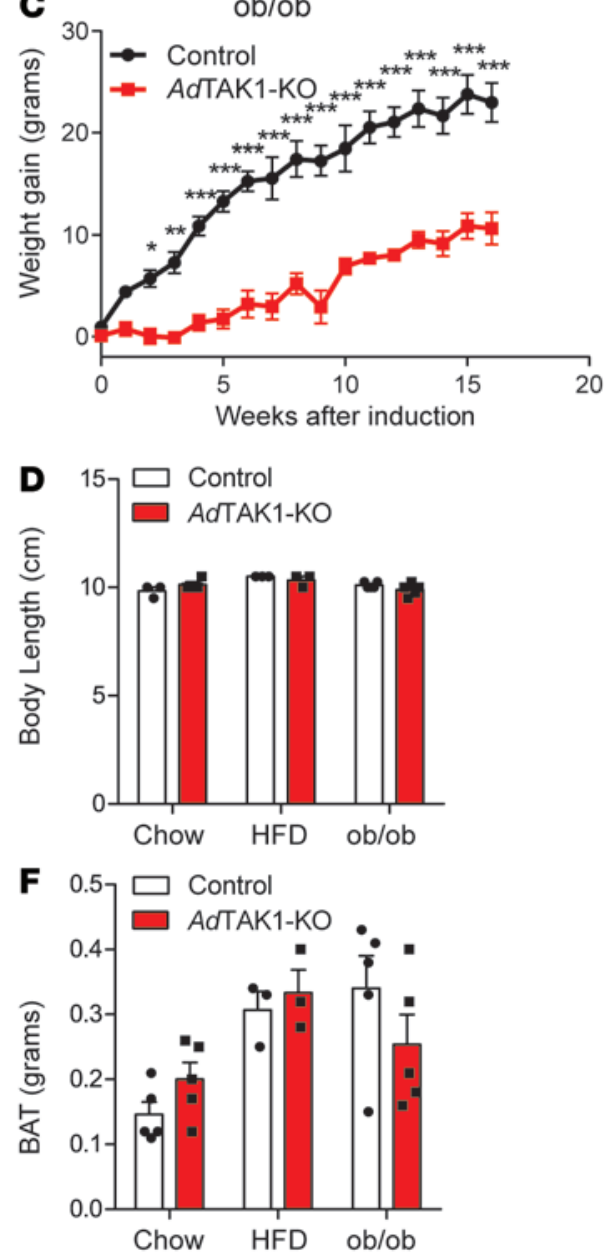
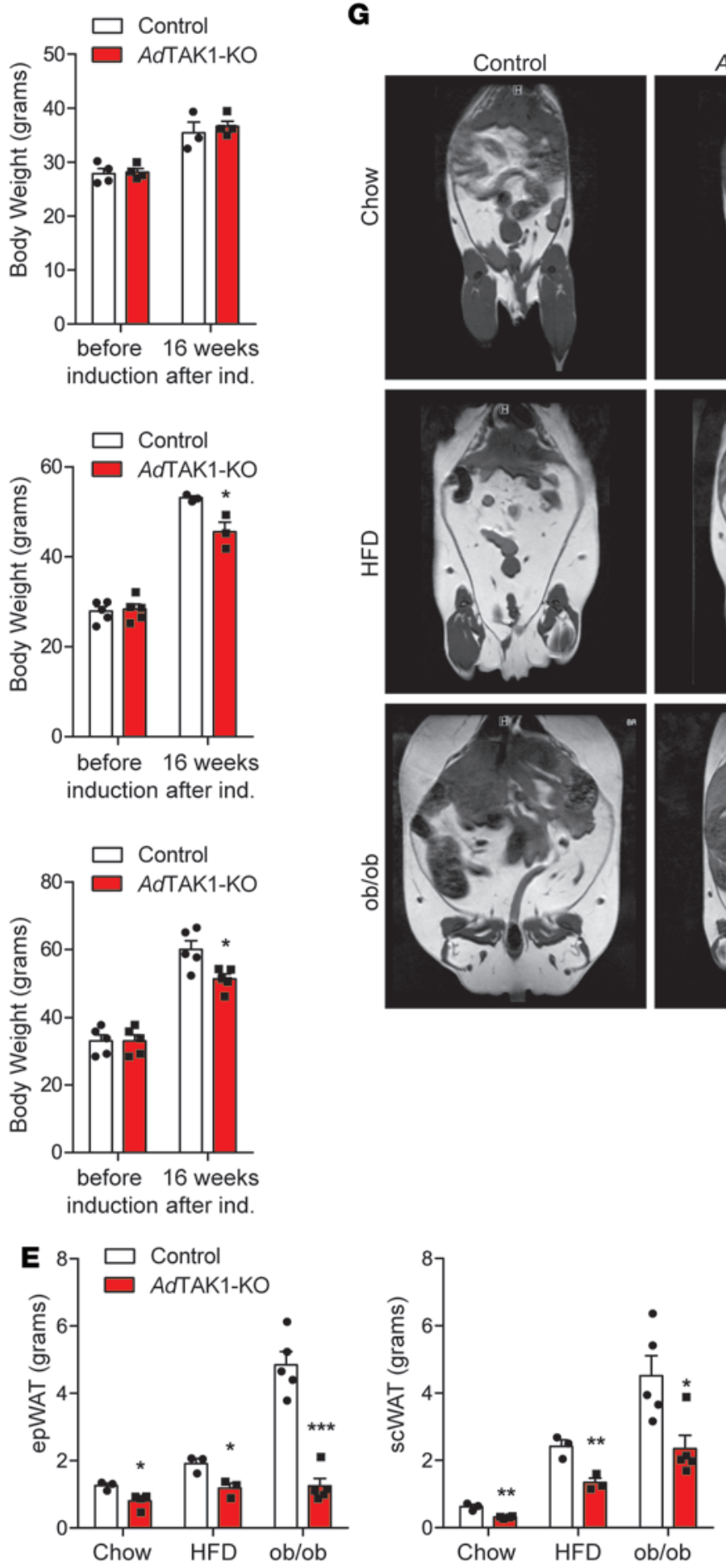

Figure 4. Reduced body weight and adipose tissue mass in AdTAK1-KO mice. (A-C) Absolute weight gain (left panels) and body weight (right panels) in AdTAK1-KO and wild-type (control) mice fed chow (A) or an HFD (B) for 16 weeks or on an ob/ob background (C) ( $n=4-5 /$ group). (D-F) Analysis of body length (D) and weight of epididymal (epWAT) or subcutaneous inguinal WAT (scWAT) (E) and of brown adipose tissue (BAT) (F) from AdTAK1-KO and wild-type (control) mice fed chow or an HFD for 16 weeks or on an $o b / o b$ background ( $n=3-5 / g r o u p)$. (G) Representative MRI images of AdTAK1-KO and wild-type (control) mice fed chow or an HFD for 16 weeks or on an $o b / o b$ background. Shown are mean values \pm SEM. ${ }^{*} P \leq 0.05$, ${ }^{*} P \leq 0.01$, ${ }^{* *} P \leq 0.001$ (ANOVA). 
A $\square$ Control
AdTAK1-KO

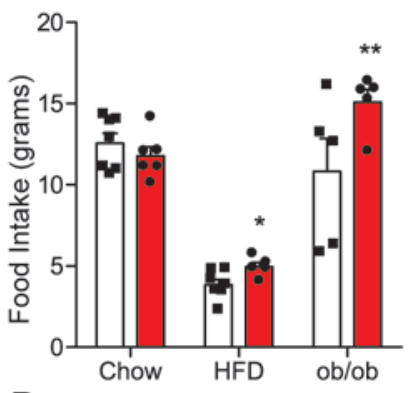

D

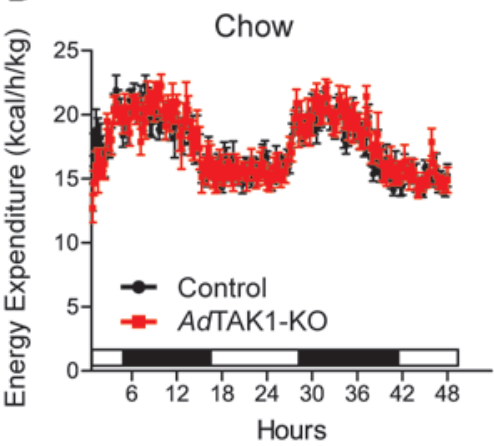

E
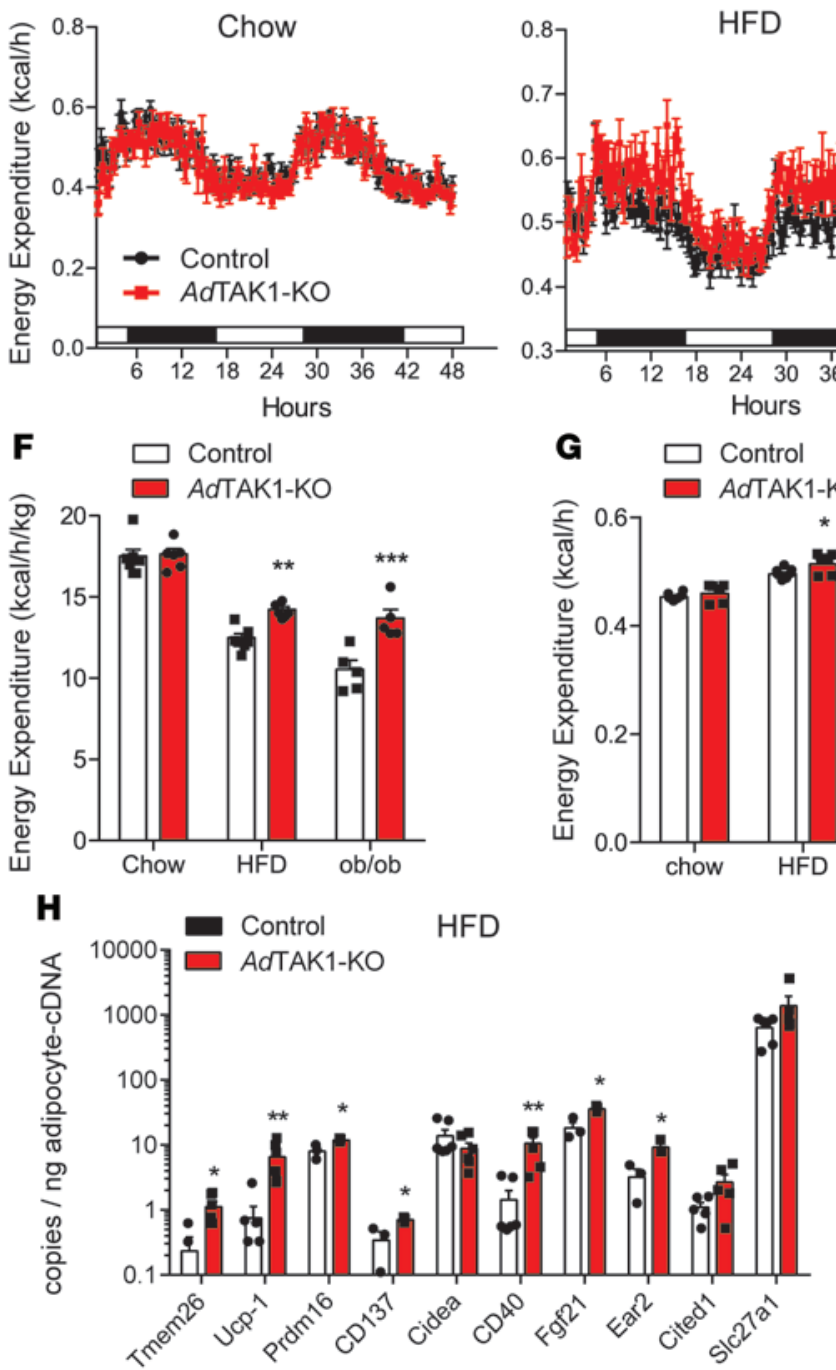

HFD

HFD

Hours

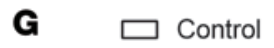

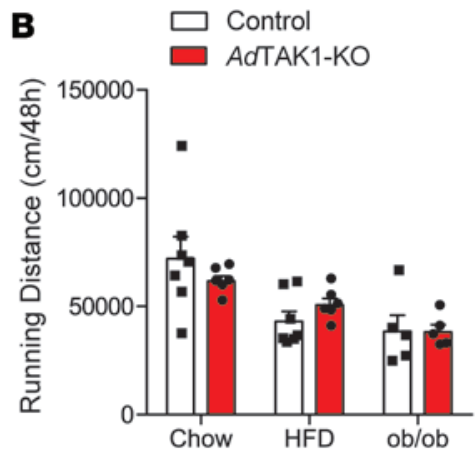
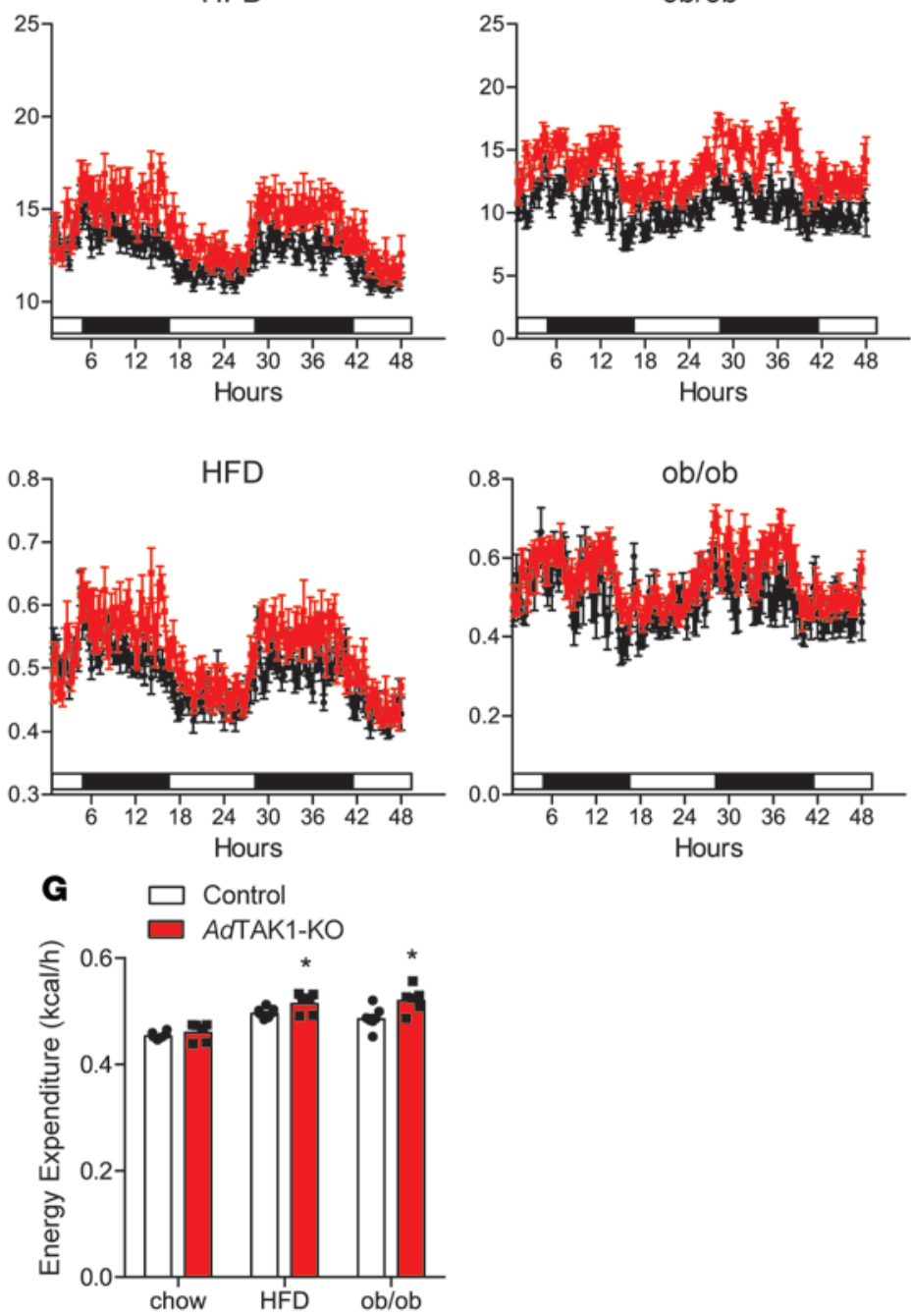

Hours

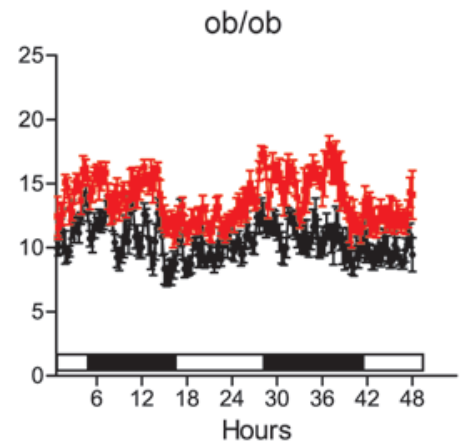

Hours

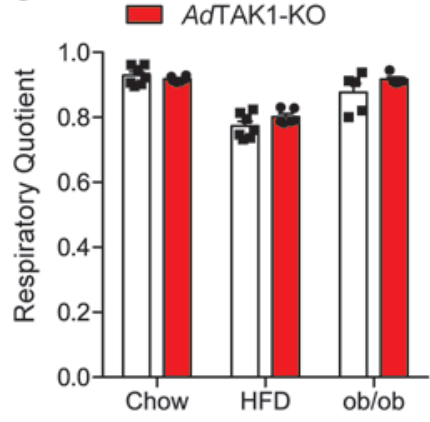

ob/ob

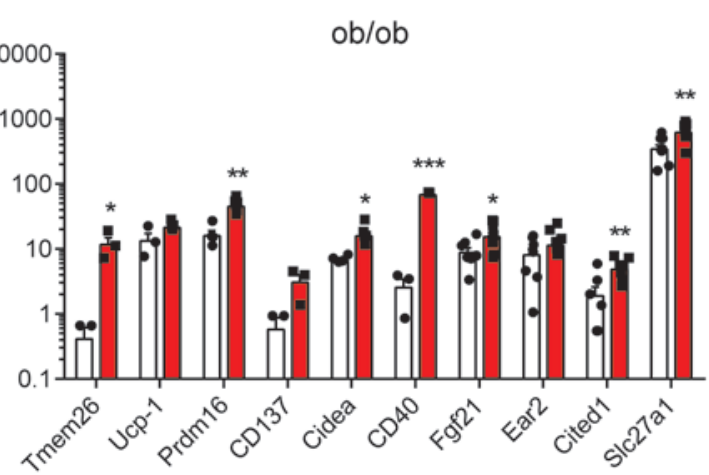


Figure 5. Analysis of metabolism and thermogenesis. (A-G) Food intake (A), activity (B), respiratory quotient (C), ratio-expressed energy expenditure (D), whole-body energy expenditure (E), average whole-body energy expenditure (F), and weight-adjusted average energy expenditure (chow: $26.3 \mathrm{~g}$; HFD: $33.0 \mathrm{~g}$, ob/ob: $38.5 \mathrm{~g}$ ) by analysis of covariance (ANCOVA) (G) in wild-type (control) and AdTAK1-KO mice fed normal diet (chow) or an HFD for 16 weeks or bred on an $o b / o b$ background ( $n=5-7 /$ group). (H) Expression of beige adipocyte marker genes in wild-type (control) and AdTAK1-KO mice fed an HFD for 16 weeks or bred on an ob/ob background ( $n=3-9$ /group). Shown are mean values \pm SEM. ${ }^{*} P \leq 0.05,{ }^{* *} P \leq 0.01,{ }^{* *} P \leq 0.001$ (ANOVA).

phages, which are characteristic of obese fat tissue (35-37). Our data are consistent with this and indicate that increased apoptosis of adipocytes during obesity development is accompanied by increased infiltration of M2 macrophages and does not lead to systemic inflammation and decreased insulin sensitivity, but rather improves glucose tolerance.

TAK1 is a central regulator of NF- $\mathrm{KB}$ activation, which is an important mediator of cell survival (20, 21). Consistent with this, loss of TAK1 has been shown to increase the sensitivity of cells for induction of apoptotic cell death $(23,25,28,38)$. In many cases, TNF- $\alpha$ has been suspected to be an upstream stimulus of TAK1-dependent cell survival, and TAK1 deficiency was shown to induce sensitivity for TNF- $\alpha$ induced cell death $(22,31,39)$. However, direct incubation of isolated TAK1-deficient adipocytes with TNF- $\alpha$ did not result in increased cell death, nor was treatment with the TNF- $\alpha$ blocker etanercept in vitro and in vivo able to reduce death of TAK1-deficient adipocytes. It is quite possible that more than one mediator that activates TAK1-dependent signaling is involved in the induction of adipocyte death in the absence of TAK1. Identifying mediators capable of inducing the death of TAK1-deficient adipocytes may be of interest in order to promote the programmed death of appropriately sensitized adipocytes under therapeutic conditions.

Interestingly, adipocyte-specific TAK1-deficient mice, when on an HFD or when crossed on an $o b / o b$ background, showed increased energy expenditure, which contributes to the protection from diet-induced obesity. This was accompanied by increased expression of UCP-1 and other markers of brown adipocytes in TAK1-deficient adipocytes from WAT, indicating an increase in the formation of beige adipocytes (40-42) in WAT from AdTAK1-KO animals. The increased "browning" of WAT may be the result of a changed adipocyte turnover in response to increased adipocyte apoptosis. It is also possible that the TAK1-mediated adipocyte signaling pathway suppresses a thermogenic gene program. Consistent with this, global deletion of $\mathrm{IKK} \varepsilon$, a downstream target of the NF- $\mathrm{B}$ pathway, has also been shown to increase energy expenditure in IKK $\varepsilon$-deficient mice and to increase UCP-1 expression in IKK $\varepsilon$-deficient WAT adipocytes (43). TAK1 is regulated by various receptors, including members of the TGF- $\beta$ receptor family. Blockade of ActRIIb, a member of the TGF- $\beta$ receptor family that is able to regulate TAK1 $(44,45)$, has been shown to increase a thermogenic gene program, including the regulation of UCP-1 in WAT and BAT $(46,47)$, suggesting an alternative mechanism by which adipocyte-specific TAK1 deficiency may increase UCP-1 expression in WAT. Our data show that increased apoptosis in TAK1-deficient adipocytes leads to infiltration of M2-polarized macrophages expressing tyrosine hydroxylase, a rate-limiting enzyme in catecholamine biosynthesis. Given the evidence for a critical role of alternatively activated macrophages and increased catecholamine production in the generation of beige fat and thermogenesis (48-51), this is also a very likely mechanism underlying the increased expression of browning markers and thermogenesis in TAK1-deficient adipocytes. We therefore think that the loss or inhibition of TAK1 in adipocytes resulting in increased apoptosis, a reduction in total number of adipocytes, and increased browning of WAT is an attractive approach to treat obesity and accompanying disorders.

We show here that increasing the rate of programmed adipocyte death resulting in a reduced number of adipocytes can prevent diet-induced obesity and prevents further weight gain under an HFD. Whereas severe obesity goes along with an increased number of adipocytes, weight reduction in obese patients does not lead to a decrease in adipocyte number, which may be a factor impeding constant weight loss. Decreasing the adipocyte number may be a strategy to treat obesity and to facilitate maintaining body weight after a weight loss.

\section{Methods}

Materials. Tamoxifen and collagenase II were from Sigma-Aldrich; antibodies against phospho-JNK (catalog 9251), JNK (catalog 9252), IкB $\alpha$ (catalog 4812), phospho-IкB $\alpha$ (catalog 9246), and TAK1 (catalog 5206) were from Cell Signaling Technology; antibodies against Mac-2 (catalog CL8942AP) were from Cedarlane; and anti- $\gamma$-tubulin antibodies (catalog T9026) were from Sigma-Aldrich. TNF- $\alpha$ was 
A

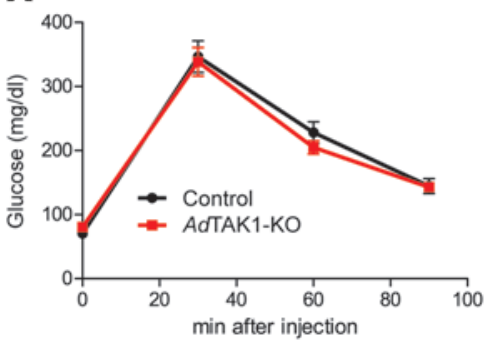

HFD

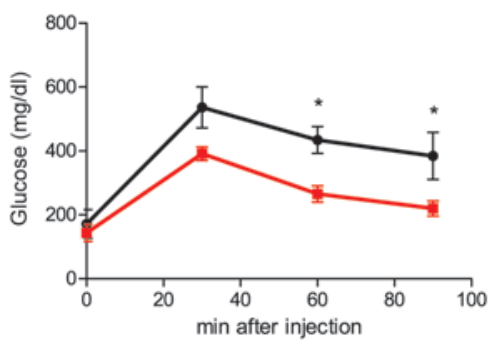

ob/ob

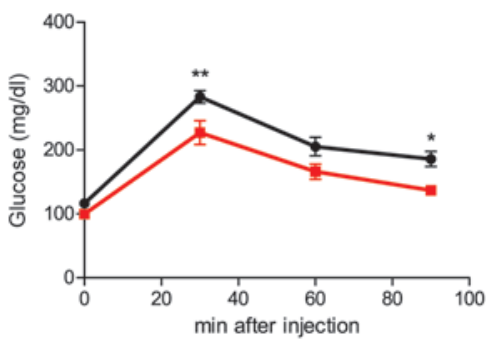

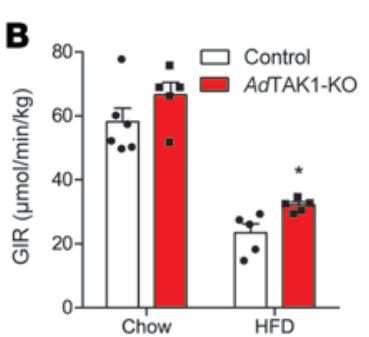
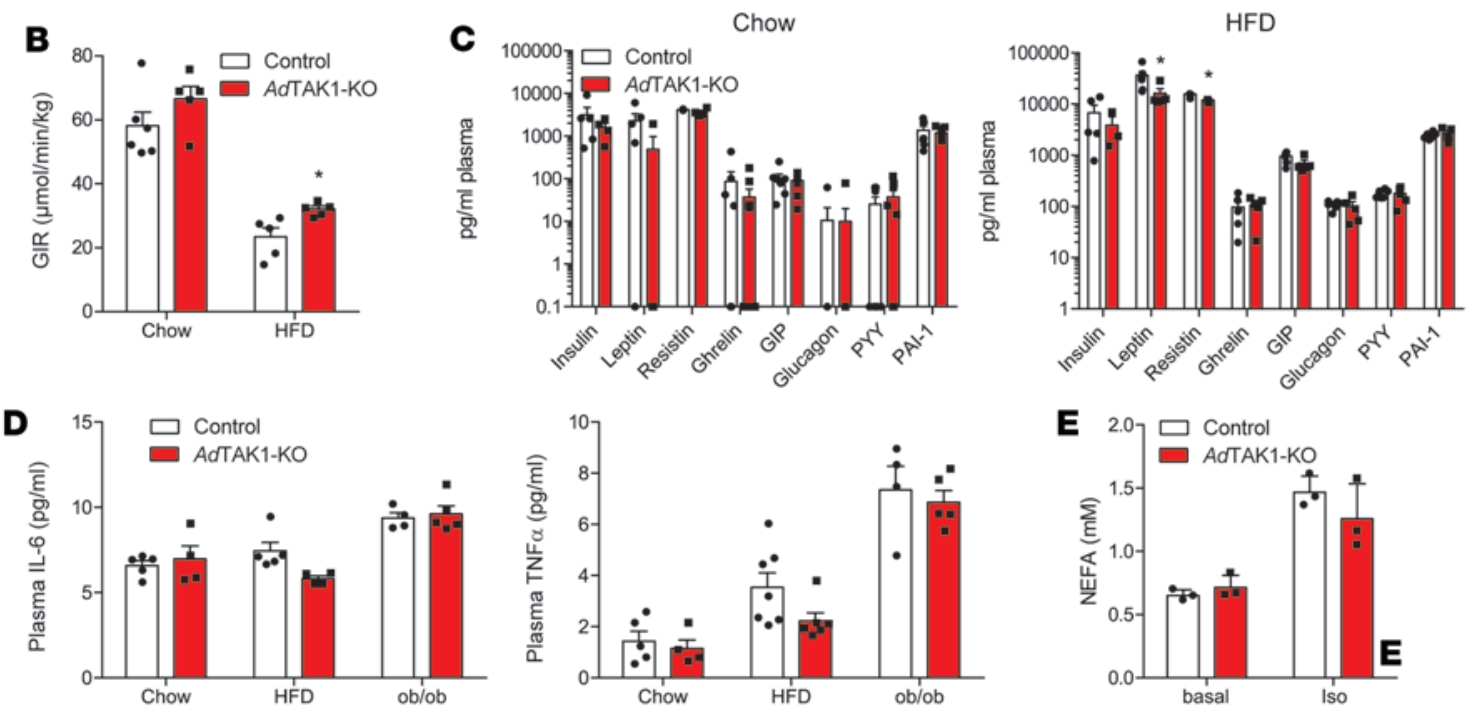

E ${ }^{2.0} 7 \square$ Control
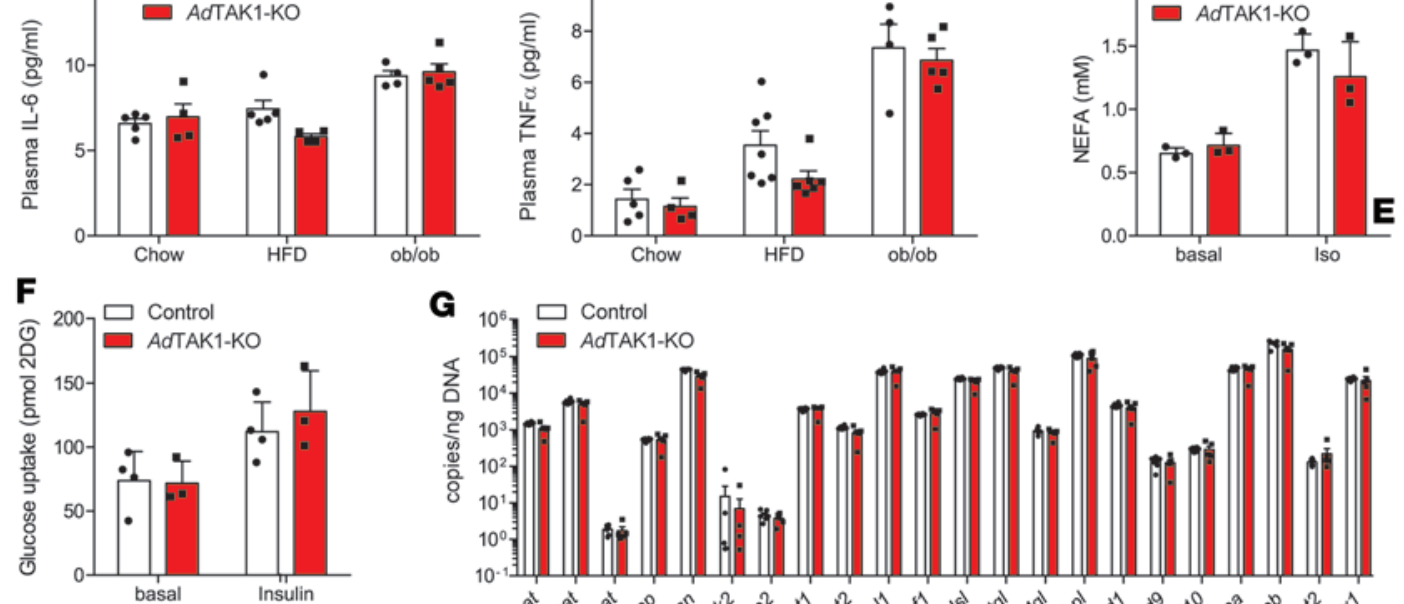

G ${ }_{10}^{6} 1 \square$ Control
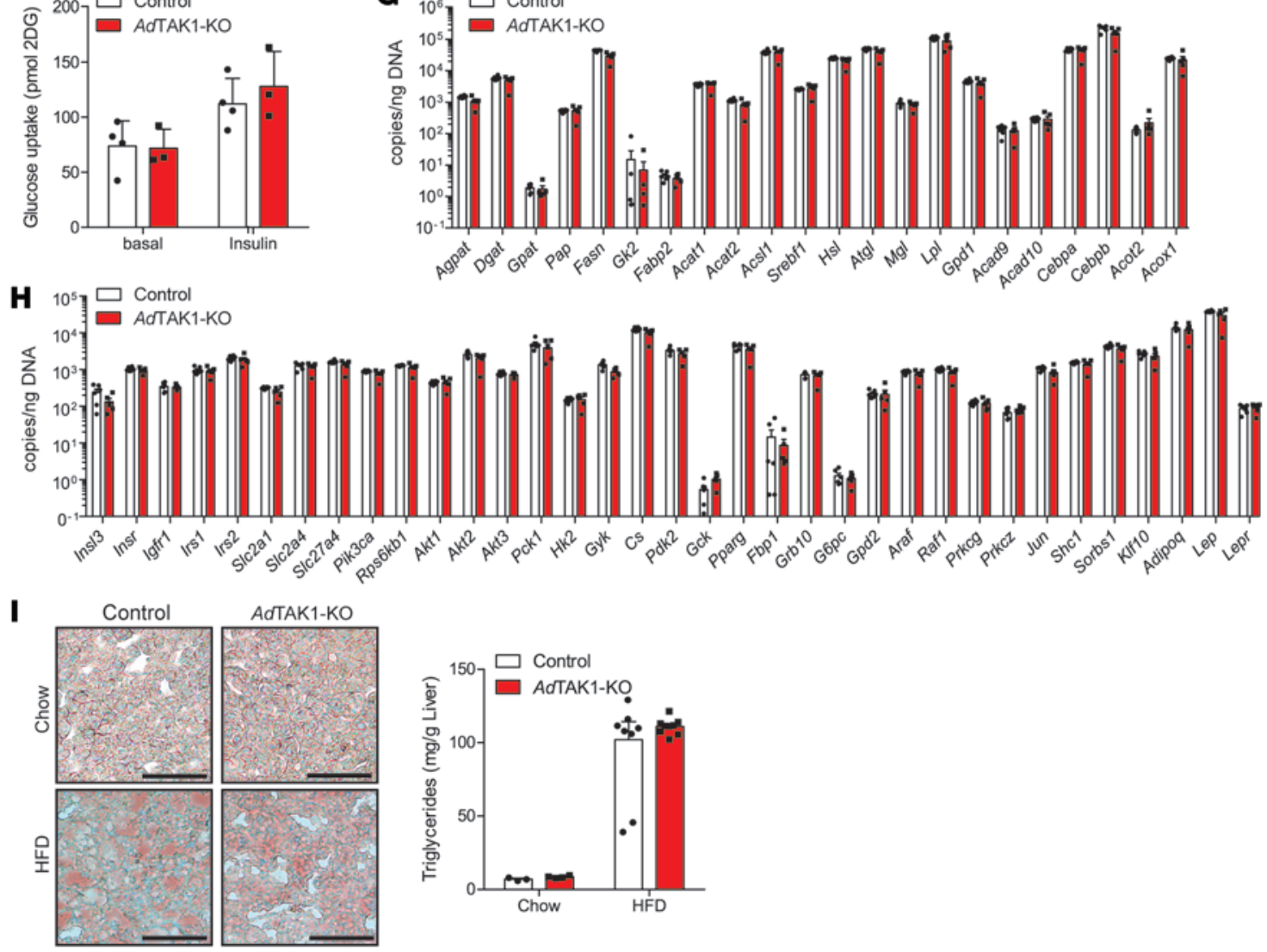
Figure 6. Glucose metabolism in AdTAK1-KO mice. (A) Intraperitoneal glucose tolerance tests were performed with wild-type (control) and AdTAK1-KO mice fed chow or an HFD for 16 weeks or on an ob/ob background ( $n=3-7 /$ group). (B) Euglycemic-hyperinsulinemic clamp studies on wild-type (control) and AdTAK1-KO mice fed chow or an HFD for 16 weeks $(n=5-6)$. (C) Plasma levels of the indicated hormones in wild-type (control) and AdTAK1-KO mice fed chow or an HFD for 16 weeks $(n=3-7)$. (D) Plasma levels of IL-6 and TNF- $\alpha$ in AdTAK1-KO and wild-type (control) mice fed chow or an HFD for 16 weeks or on an ob/ob background ( $n=4-6 /$ group). (E) Lipolytic activity of wild-type (control) and AdTAK1-KO epididymal adipocytes under basal conditions and after stimulation with $1 \mu \mathrm{M}$ isoproterenol (Iso) ( $n=3 /$ group). (F) Glucose uptake of wild-type (control) and AdTAK1-KO epididymal adipocytes under basal conditions and after stimulation with $1 \mu \mathrm{M}$ insulin ( $n=3-4 /$ group). (C) Quantitative PCR showing expression levels of genes regulating lipid metabolism ( $n=5-6 /$ group). (H) Quantitative PCR showing expression levels of genes regulating glucose metabolism ( $n=5-6 / g r o u p$ ). (I) Oil Red 0 staining of liver sections from wild-type (control) and AdTAK1-KO mice fed chow or an HFD for 16 weeks (scale bars: $100 \mu \mathrm{m}$ ) and liver triglyceride content per gram liver tissue in control and AdTAK1-KO mice fed chow or an HFD for 16 weeks ( $n=3-9 / g r o u p)$. Shown are mean values \pm SEM. ${ }^{*} P \leq 0.05,{ }^{* *} P \leq 0.01$ (ANOVA).

from R\&D Systems; etanercept (Enbrel) was from Pfizer; and Ac-DEVD-AMC and Z-VAD-FMK were from Enzo Life Sciences.

Experimental animals. Adipoq $\mathrm{CreER}^{\mathrm{T} 2}$ mice (29) were crossed with $\mathrm{TAK} 1^{\mathrm{1} / \mathrm{l}}$ (30) mice to generate adipocyte-specific tamoxifen-inducible TAK1-KO mice (AdipoqCreER $\left.{ }^{\mathrm{T} 2+/} ; \mathrm{TAK}^{\mathrm{f} / \mathrm{f}}\right)$. AdipoqCreER ${ }^{\mathrm{T} 2}$ negative littermates were used as control (AdipoqCreER $\left.{ }^{\mathrm{T} 2-/-} ; \mathrm{TAK} 1^{\mathrm{H} / \mathrm{f}}\right)$. Animals were backcrossed on a C57BL/6 background and housed under specific pathogen-free conditions. To induce recombination, $1 \mathrm{mg}$ tamoxifen was injected i.p. on 5 consecutive days. To validate Cre-mediated recombination, genomic DNA was isolated from adipocytes, SVF, and BAT of AdipoqCreER ${ }^{\mathrm{T} 2+-} ; \mathrm{TAK}^{\mathrm{H} / \mathrm{fl}}$ and control animals using the DNeasy Blood \& Tissue Kit (QIAGEN). Quantitative real-time PCR was performed using Light Cycler 480 SYBR Green I and the LightCycler 480 II (all Roche Applied Sciences). Genomic DNA from mouse tissue was used for quantification. Primers for detection of the TAK1 allele were 5'-TTCTGATTTTTGGGGGTTTTC-3' and 5'-CAATGAAAGCCTTCCTCTCAG-3'.

Mice were fed a standard pellet chow (Altromin) or an HFD containing $30 \%$ fat (ssniff) with water ad libitum.

WAT explant culture and TNF- $\alpha$ stimulation. WAT explants of approximately $20 \mathrm{mg}$ from epididymal fat pads were incubated in DMEM supplemented with penicillin and streptomycin (both $10 \mathrm{U} / \mathrm{ml}$ ) at $37^{\circ} \mathrm{C}$ and $5 \% \mathrm{CO}_{2}$ for 24 hours; thereafter, $20 \mathrm{ng} / \mathrm{ml} \mathrm{TNF}-\alpha$ was added for the indicated time points, and tissue explants were snap-frozen in liquid nitrogen.

Western blot. Samples were prepared by homogenizing adipocytes or adipose tissue in lysis buffer (10 mM Tris pH 8.0, $130 \mathrm{mM} \mathrm{NaCl}, 1 \%$ Triton X-100, $10 \mathrm{mM} \mathrm{NaF}, 10 \mathrm{mM} \mathrm{NaPi}$ [pH 7.5], $10 \mathrm{mM} \mathrm{NaPPi}$ [pH 7.5]) using a dounce homogenizer. Samples were incubated on ice for 60 minutes and then centrifuged at $4^{\circ} \mathrm{C}$ for 30 minutes at $20,000 \mathrm{~g}$. Protein concentration of the supernatants was determined, and samples were diluted with $4 \times$ Läemmli buffer to a final protein concentration of $1 \mathrm{mg} / \mathrm{ml}$ and heated to $95^{\circ} \mathrm{C}$ for 5 minutes.

For detection of TAK1 in adipocytes, samples were washed with PBS and 1× Cell Lysis Buffer (Cell Signaling Technology) was added. Samples were sonicated 3 times 15 seconds each and centrifuged for 10 minutes at $4^{\circ} \mathrm{C}$ and $20,000 \mathrm{~g}$. The supernatant was transferred to a new tube, protein concentration was determined, and samples were diluted to a final protein concentration of $1 \mathrm{mg} / \mathrm{ml}$. Primary antibody directed against TAK1 (Cell Signaling Technology, catalog 5206) was added to the cell lysate, and samples were incubated overnight at $4^{\circ} \mathrm{C}$ with rotation. Protein A magnetic beads (Cell Signaling Technology) were added and incubated for 30 minutes at $4^{\circ} \mathrm{C}$ with rotation. Beads were pelleted using a magnetic separation rack, and pellets were washed five times and analyzed by Western blotting.

Isolation of adipocytes and $S V F$. Adipocytes from mouse epididymal fat pads were isolated according to a modified method described by Rodbell (52). Briefly, adipocytes were digested with collagenase II ( $0.25 \mathrm{mg}$ / $\mathrm{ml}$ ) in a buffer containing $125 \mathrm{mM} \mathrm{NaCl}, 5 \mathrm{mM} \mathrm{KCl}, 1 \mathrm{mM} \mathrm{CaCl}_{2}, 2.5 \mathrm{mM} \mathrm{MgCl}_{2}, 1 \mathrm{mM} \mathrm{KH}_{2} \mathrm{PO}_{4}, 2 \%$ BSA, $4 \mathrm{mM}$ glucose, and $25 \mathrm{mM}$ Tris (pH 7.4) for 1 hour at $37^{\circ} \mathrm{C}$. Cells were filtered through a nylon mesh and washed three times with the same buffer. Used wash buffer was collected and centrifuged for 5 minutes at $250 \mathrm{~g}$ to collect the SVF. Isolated adipocytes were cultured at $37^{\circ} \mathrm{C}$ and $5 \% \mathrm{CO}_{2}$ in DMEM/FBS/antibiotic solution. Incubation with $1 \mu \mathrm{M}$ 4-OH-tamoxifen (Sigma-Aldrich) was for 72 hours.

Caspase-3 activity assay. WAT or isolated adipocytes were homogenized in a dounce homogenizer in lysis buffer $\left(10 \mathrm{mM}\right.$ Tris- $\mathrm{HCl}, 10 \mathrm{mM} \mathrm{NaH} \mathrm{PO}_{4} / \mathrm{NaHPO}_{4}, \mathrm{pH} 7.5,130 \mathrm{mM} \mathrm{NaCl}, 1 \%$ Triton X-100, 10 $\mathrm{mM} \mathrm{NaPPi}$ ) and kept on ice for 30 minutes. Samples were centrifuged at $4^{\circ} \mathrm{C}$ and 20,000 $\mathrm{g}$ for 15 minutes and supernatants taken for caspase- 3 activity assay and protein concentration determination. For caspase- 3 

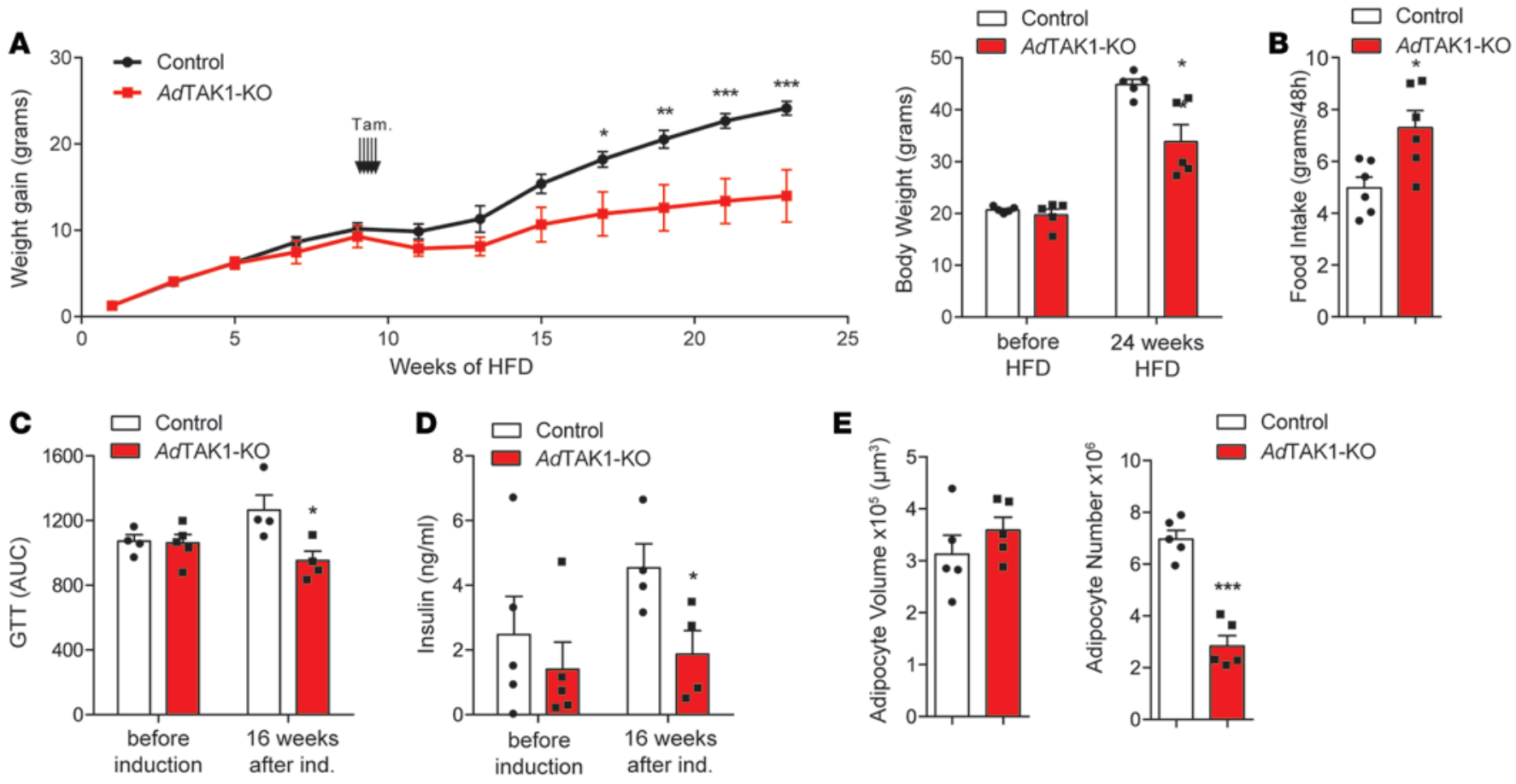

Figure 7. Effects of induction of adipocyte-specific TAK1 deficiency in mice with established obesity due to HFD. (A) Absolute body weight gain in HFD-fed AdTAK1-KO and wild-type mice (control) 8 weeks before and 16 weeks after induction with tamoxifen ( $n=5 /$ group). The bar diagram shows the statistical analysis of the body weight at the beginning and after 24 weeks of an HFD. (B) Food intake over 48 hours, 16 weeks after tamoxifen induction ( $n=6$ ). (C) Intraperitoneal glucose tolerance test before and 16 weeks after tamoxifen induction $(n=4-5)$. (D) Basal plasma insulin before and 16 weeks after tamoxifen induction $(n=4-5)$. (E) Average adipocyte volume and adipocyte number in epididymal adipose tissue 16 weeks after tamoxifen induction of $A d T A K 1-K O$ and wild-type (control) mice $(n=5)$. Shown are mean values \pm SEM. ${ }^{*} P \leq 0.05,{ }^{* *} P \leq 0.01,{ }^{* * *} P \leq 0.001$ (ANOVA [A, C, and $\left.\mathbf{D}\right]$ or $t$ test [B and $\mathbf{E}$ ).

activity assays, $20 \mu 1$ sample and $200 \mu 1$ assay buffer (20 mM HEPES [pH 7.5], 10\% glycerol, 2 mM DTT, $20 \mu \mathrm{M}$ Ac-DEVD-AMC) were incubated at $37^{\circ} \mathrm{C}$ for 2 hours and luminescence was measured (FlexStation 3, Molecular Devices).

Glucose uptake assay. Glucose uptake of freshly isolated epididymal white adipocytes was measured using the Glucose Uptake Colorimetric Assay Kit (Sigma-Aldrich) according to the manufacturer's instructions. Fifty microliters of adipocytes were placed in each well of a 96-well plate in $100 \mu \mathrm{l}$ buffer without glucose and incubated at $37^{\circ} \mathrm{C}$ for 1 hour, followed by stimulation with or without $1 \mu \mathrm{M}$ insulin in the presence of $1 \mathrm{mM} 2$-deoxyglucose for 30 minutes.

Lipolysis assay. One hundred microliters of freshly isolated epididymal white adipocytes were incubated with or without $1 \mu \mathrm{M}$ isoproterenol for 2 hours at $37^{\circ} \mathrm{C}$. Lipolytic activity was determined by measurement of nonesterified free fatty acid release using the NEFA Non Esterified Fatty Acids kit (RANDOX Laboratories Ltd.).

Quantitative real-time PCR. RNA was isolated from adipocytes of Adipoq $\mathrm{CreER}^{\mathrm{T} 2+/} ; \mathrm{TAK} 1^{\mathrm{H} / \mathrm{l}}$ and control animals using the RNeasy Lipid Tissue Mini Kit (QIAGEN). Remaining genomic DNA was removed using the TURBO DNA-free Kit (Life Technologies), and cDNA synthesis was performed by using the Transcriptor First Strand cDNA Synthesis Kit (Roche Applied Science). Quantitative real-time PCR was performed using the Universal ProbeLibrary, LightCycler 480 Probes Master, and the LightCycler 480 II (all Roche Applied Sciences). Genomic DNA from mouse tissue was used for quantification. Primer sequences were: CCL22 5'-CATCCACGTGTTGGCTCA-3' and 5'-GGATCATCTTGCTGGTGAATG-3' probe 62; IL10 5'-AGCCGGGAAGACAATAACTG-3' and 5'-GCAGTCCGCAGCTCTAGG-3' probe 21; IL1rn 5'- TGGAAGTAGCTATGTTCCTGACTG-3' and 5'-CACACGAAGCTCCCTGATCT-3' probe 53; Chi313 5'-AAGAACACTGAGCTAAAAACTCTCCT-3' and 5'-GAGACCATGGCACTGAACG-3' probe 88; Itgax 5'-GAGTCAGGGCCTCTGGAGTA-3' and 5'-GTGAGCCTCCACGGAAAAT-3' probe 84; Arg1 5'-GGCCTTTGTTGATGTCCCTA-3' and 5'-ACAGACCGTGGGTTCTTCAC-3' probe 17; IL12b 5'-CGCAGCAAAGCAAGGTAAGT-3' and 5'-CCTCTAGATGCAGGGAGTTAGC-3' probe 103; Cxc19 5'-TGCTAGAGGCAAAAACTCTGTG-3' and 5'-TAGGCTCAAGGGCGTGAT-3' probe 76; 
Cxcl11 5'-CTGTGAATGAATGGTAGGGATG-3' and 5'-GAAACACAATTCTTATTCTTTTCAAGG-3' probe 21; IL1b 5'-CAAAAGATGAAGGGCTGCTT-3' and 5'-GAAGCTGGATGCTCTCATCA-3' probe 26; Cxc110 5'-CCTTGGTCTTCTGAAAGGTGA-3' and 5'-CATGGCTTGACCATCATCC-3' probe 63; Nos2 5'-AAGGGGACGAACTCAGTGG-3' and 5'-CCCGGAAGGTTTGTACAGC-3' probe 88; CD68 5'-TTCTGCTGTGGAAATGCAAG-3' and 5'-AGAGAGAGCAGGTCAAGGTGA-3' probe 78; Emr1 5'-CAGCTCTCGCAACATCAAGA-3' and 5'-AGGACTGGAAGCCCATAGC-3' probe 98; CD11b 5'-TCTGGCAGATGTGGCTATTG-3' and 5'-GTCCTGTCTTGAGGCTCCAT-3' probe 55; Tmem26 5'-GTGAACCGGTCTGGACCTT-3' and 5'-AAGCCACCGTCCAATGATTA-3' probe 22; Ucp 1 5'-CGTGAAGGTCAGAATGCAAG-3' and 5'-GCATTGTAGGTCCCCGTGTA-3' probe 21; Prdm16 5'-GAGGACTTCACTCCCAAGGA-3' and 5'-GATTGGAATGTCTTCAGGAATGT-3' probe 92; CD137 5'-CAAGAGCTGCCCTCCAAGTA-3' and 5'-CAGATGTTACAGTTCGGCTGTC-3' probe 67; Cidea 5'-GCAAGCAACCAAAGAAATCG-3' and 5'-AGGAAGTCCTTGGGGTTCAG-3' probe 106; CD40 5'-AGCCACTGCACAGCTCTTG-3' and 5'-GTGCTGGTGACAGCGAATC-3' probe 26; Fgf21 5'-ACTGTGGGTCCGACTGCT-3' and 5'-GGATGGGGTATGCTTGGTAG-3' probe 3; Ear2 5'-TCATAATAGTTCATCTCGGGTACG-3' and 5'-TTGGTTGGTATCTGCATTGG-3' probe 9; Mrc1 5'-GTGGACTGTGGACGAGCAG-3' and 5'-CCTTTCGTCCTGGCATGT-3' probe 47; Th 5'-GAGTACTTTGTGCGTTCGAG-3' and 5'-CGACGCACAGAACTGAGG-3' probe 71; Cited1 5'-AGGGTCTCCGGGATCTGT-3' and 5'-GGGTCAGAATCAATGAGAGCA-3' probe 63; Slc27a1 5'-CTTTGCACCAGGCGATGT-3' and 5'-GCCACAGTCCCACGAACT-3' probe 76; Ins13 5'-GCAGTGGCTAGAGCAGAGACA-3' and 5'-AGAGGAAGCTGCGGGTCTA-3' probe 49; Insr 5'-CCCTGGTTATCTTCGAGATGG-3' and 5'-CGGGTGATGTTCATGAGGTT-3' probe 17; Igf1r 5'-GGAATGTGGGGACCTGTGT-3' and 5'-TTGTTGATGGTGGTCTTCTCA-3' probe 25; Irs1 5'-GCAGGCACCATCTCAACAA-3' and 5'-TTCGAGATCTCCGAGTCAGTC-3' probe 20; Irs2 5'-GGTTTCATGTCCCTTGACGA-3' and 5'-CTGTGGCTACTGAAGGCTCTC-3' probe 19; Slc2a1 5'-CCATGTATGTGGGAGAGGTGT-3' and 5'-GCAATAAGGATGCCAACGAC-3' probe 33; Slc2a4 5'-ACTGCTTCTGGCTCTCACAGT-3' and 5'-GACAGAAGGGCAGCAGAATC-3' probe 108; Slc27a4 5'-GGCACAGACACTCACTGGAC-3' and 5'-GAAGTTGGCCACACTACTGGA-3' probe 42; Pik3ca 5'-TGTGTCTTAGAATATCAGGGCAAG-3' and 5'-CAGAGGGTACTTTTCCAGGAAGTAT-3' probe 5; Rps6kb1 5'-ACGAAAGCAGAGCGGAAC-3' and 5'-CCGGTCTGAAAGGCATAAAT-3' probe 69; Akt1 5'-AAGCTGTTCGAGCTGATCCT-3' and 5'-TGTGTAGGGTCCTTCTTGAGC-3' probe 25; Akt2 5'-CGCCTCTTTGAGCTCATTCT-3' and 5'-TGGGTCCTTCTTCAGCAGTC-3' probe 25; Akt3 5'-AAGCTATCCAAGCCGTAGCC-3' and 5'-GGTTGGGCTACAATTCATCC-3' probe 101; Pck1 5'-CATGACTCGGATGGGCATA-3' and 5'-CACTTGATGAACTCCCCATCT-3' probe 67; Hk2 5'-TGCCAAGCGTCTCCATAAG-3' and 5'-AGGAAGCGGACATCACAATC-3' probe 75; Gyk 5'-CCTCCATGCCTGAAACAACT-3' and 5'-CACACACCAACCCCCTCT-3' probe 45; Cs 5'-GCGTTGCAGCCAAGATCTAC-3' and 5'-TGTTGGTAAAATTGTGTGACCAG-3' probe 74; Pdk2 5'-CTGGTCACCATCCGGAAC-3' and 5'-ACGGGGTCATCTCCATAGG-3' probe 67; Gck 5'-CCTGTGAAAGCGTGTCCA-3' and 5'-GTCCTCACTGCGGCTTTC-3' probe 18; Pparg 5'-CCATTTGTCATCTACGACATGAAT-3' and 5'-GGGTGATATGTTTGAACTTGATTTT-3' probe 7; Fbp 1 5'-ACGGATATCAGCACCCTGAC-3' and 5'-TTCAGCAGCTGGGTCAACT-3' probe 4; Grb10 5'-GTGAAGATGGGACCAGCAA-3' and 5'-ACAGGTCCCTGGCTGTCAT-3' probe 22; G6pc 5'-CTTTCCCATCTGGTTCCATC-3' and 5'-TGAACCAGTCTCCGACCACT-3' probe 82; Gpd2 5'-TCACTGCTGCCAGGTACG-3' and 5'-TCAGGGTCTGTCTTCTTGAGC-3' probe 10; Araf 5'-CTTCAGCCTGGCCTTCTGT-3' and 5'-AGGAACAATGCTGGTGGAAC-3' probe 29; Raf1 5'-CAGCCCTTCCTCAGAAGGTT-3' and 5'-ACCATGTGGACATTGGGAGT-3' probe 34 ; Prkcg 5'-GGACCTCAAGTTGGATAATGTGA-3' and 5'-GCCAAAGTCTGTGATCTTGATG-3' probe 89; Prkcz 5'-ATCGGGCGTGGAAGCTAT-3' and 5'-TGGCGTAAATCTGGTCGTTT-3' probe 79; Jun 5'-TGATCATCCAGTCCAGCAAT-3' and 5'-TTGGGGCACAAGAACTGG-3' probe 9; Shc1 5'-CTTTGATGGCTCAGCTTGG-3' and 5'-CCTTCCCGAAGCCTCATATC-3' probe 69; Sorbs1 $5^{\prime}$-CCTGCCTCAGAGAGAAGAGC-3' and $5^{\prime}$-GGCGAAGTGAGTTCTGCTG-3' probe 15 ; Klf10 5'-CACCCCTGCCTGTCATCT-3' and 5'-GCTGGGGACAACTGTGCTA-3' probe 4; Adipoq 5'-CAGGCATCCCAGGACATC-3' and 5'-GCATCTCCTTTCTCTCCCTTC-3' probe 53; Lep 5'-CCCATTCTGAGTTTGTCCAAG-3' and 5'-GCTGGTGAGGACCTGTTGAT-3' probe 93; Lepr 5'-CACAACCGATGACTCCTTTCT-3' and 5'-CTTCAGAAGCCCCCTTCAA-3' probe 62; Agpat 
5'-TTGCCAAGCGTGAGCTACTAT-3' and 5'-TCCGGTCAATGAAGATGATTC-3' probe 3; Dgat 5'-AACCTGGCCACAATCATCTG-3' and 5'-GGAGTGATAGACTCAACCAGTAAGG-3' probe 3; Gpat 5'-GGTCAGCCTCTGCTCATCTT-3v and 5'-CGCCTGGATTACCACTCCTA-3' probe 81; Pap 5'-TTGCAATTCTTACTTCAAGGCATA-3' and 5'-GGGTACTTGATGGAGTCATCATT-3' probe 11; Fasn 5'-GGCTGCAGGTGGTCGATA-3' and 5'-AATGAGTTGATGCCCACGTT-3' probe 96; Gk2 5'-GCCTTTTCGGGGCTGTAT-3' and 5'-GACCACAGATTATCCCTCTTGC-3' probe 48; Fabp2 5'-AAACTTATTGGGAAATTCACACG-3' and 5'-TTACCAGAAACCTCTCGGACA-3' probe 17; Acat1 5'-AGCATTCAGTGTGGTTGTGC-3' and 5'-GGATATTTACTTTTTGGGGGTCA-3' probe 67; Acat2 5'-TCTTGCAGCCCAGTCCATA-3' and 5'-ATTCTCCATGCCTCCAGCTA-3' probe 22; Acs11 5'-ATGGACTCCTACGGCAGTGA-3' and 5'-TTTGAGGCTGATGATTTCCA-3' probe 31; Srebf1 5'-GCACACCCAGGTCCAAAG-3' and 5'-TGAGGTCACAGTGTTTGTTCTAGG-3' probe 3; Hsl 5'-CTAATGTCTTTGCAGGTGTTCG-3' and 5'-ACAGTTGGCCTAGGGTTGGT-3' probe 45; Atgl 5'-ACCATCCGTGGCTGTCTACT-3' and 5'-AACACGAGTCAGGGAGATGC-3' probe 40; $\mathrm{Mgl}$ 5'-TGGCCTGAAGCTGACAAGTA-3' and 5'-CCTCCAGGGAGTTGACCAC-3' probe 19; Lpl 5'-CCAAGGAAGCCTTTGAGAAA-3' and 5'-CCCAGATTGTTACAGCGATTC-3' probe 13; Gpd1 5'-GCCAAGCTCTTCTGCAGTG-3' and 5'-CATAGCAGGTCGTGATGAGG-3' probe 80; Acad9 5'-TCTGGCTGTGTGCTGCTC-3' and 5'-CCGTGAATTCCCTCAGCA-3' probe 70; Acad10 5'-TGCAGTCCTCACCAACAACT-3' and 5'-ATCAAACTGTTTCCGGTCCA-3' probe 4; Cebpa 5'-AAACAACGCAACGTGGAGA-3' and 5'-GCGGTCATTGTCACTGGTC-3' probe 67; Cebpb 5'-ATCGACTTCAGCCCCTACCT-3' and 5'-TAGTCGTCGGCGAAGAGG-3' probe 55; Acot2 5'-GAGGTGGCCTTCTGGAGTATC-3' and 5'-GGGGAGGTCGTCGTAGTTG-3' probe 21; Acox1 5'-CACTCGAAGCCAGCGTTAC-3' and 5'-GCCAAATTCCCTCATCTTCTT-3' probe 83.

Histology and immunohistochemistry. H\&E staining was performed on $4-\mu \mathrm{m}$ paraffin sections, and Oil Red O staining was performed on cryosections according to standard protocols. For immunohistochemical staining of adipose tissue sections, $18-\mu \mathrm{m}$ cryosections were treated according to the instructions of the In Situ Cell Death Detection Kit (Roche Applied Sciences). After washing twice in PBS, slides were incubated for 10 minutes with DAPI (Life Technologies), and finally mounted in Mowiol (Calbiochem/EMD Biosciences) and analyzed on a Leica TCS SP5 confocal microscope by an experimenter blinded to genotype.

Mac-2 staining was performed on 4- $\mu \mathrm{m}$ paraffin-embedded adipose tissue sections. Sections were deparaffinized and incubated in sodium citrate buffer at $95^{\circ} \mathrm{C}$ for 30 minutes. Sections were washed in PBST, incubated in $0.3 \% \mathrm{H}_{2} \mathrm{O}_{2}$ for 30 minutes, and washed in PBS. Normal goat serum was used for blocking for 20 minutes at room temperature, and incubation with Mac-2 antibody (Cedarlane, catalog CL8942AP) was performed at $4^{\circ} \mathrm{C}$ for 24 hours. Slides were washed and secondary antibody labeling was performed using the Vectastain Elite ABC Kit and DAB Peroxidase Substrate (both from Vector Laboratories).

Using ImageJ software (NIH), adipocyte volume was calculated from H\&E-stained paraffin sections as described previously (53). Adipocyte number was calculated by dividing the epididymal adipose tissue volume (calculated by multiplying the epididymal adipose tissue weight and commonly used adipose tissue density factor $0.92 \mathrm{~g} / \mathrm{cm}^{3}$ ) by adipocyte volume (54).

Flow cytometry and BrdU labeling. Isolated cells of the SVF were washed in PBS and 3\% heat-inactivated FBS and filtered through a 40- $\mu$ m cell filter. Cells $\left(1 \times 10^{6}\right)$ were incubated with Fixable Viability Dye eFluor660 (eBioscience) and antibodies directed against F4/80 (eBioscience, catalog 47-4801), CD43 (BD Biosciences, catalog 553269), Pref-1 (MBL International Corporation, catalog D187-5), or BrdU (BD Biosciences, catalog 556028) and analyzed by flow cytometry using the BD FACSCanto II.

One milligram of BrdU (BD Biosciences) was injected i.p. on 10 consecutive days after 2 weeks of HFD feeding. SVF cells were isolated from epididymal fat pads and analyzed by flow cytometry using the FITC BrdU Flow Kit (BD Biosciences).

Plasma TNF- $\alpha$, IL-6, insulin, resistin, and leptin. Blood samples were collected from the retro-orbital plexus or the tail vein and in EDTA (10 mM final concentration). Plasma levels of TNF- $\alpha$ and IL- 6 were determined using mouse TNF- $\alpha$ and IL-6 ELISA (eBioscience), plasma levels of insulin and resistin were measured using rat/mouse insulin and mouse resistin ELISA kits (Millipore), and plasma levels of leptin were determined using a leptin (mouse/rat) ELISA kit (Enzo Life Sciences).

Plasma ghrelin, GIP, glucagon, PYY, and PAI-1. Blood samples were collected from the retro-orbital plexus or the tail vein and in EDTA (10 mM final concentration). Plasma levels were determined using the MAGPIX system combined with MILLIPLEX MAP magnetic bead-based multi-analyte panels and MILLI- 
PLEX Analyst 5.1 software (Merck Millipore).

Magnetic resonance imaging. All MRI experiments were performed on a 7.0T superconducting magnet (Bruker Biospin, Pharmascan, 70/16, $16 \mathrm{~cm}$ ) equipped with an actively shielded imaging gradient field of $300 \mathrm{mT} / \mathrm{m}$. The frequency for the ${ }^{1} \mathrm{H}$ isotope is $300.33 \mathrm{MHz}$. A $60-\mathrm{mm}$ inner diameter linearly polarized ${ }^{1} \mathrm{H}$ volume resonator was used for RF pulse transmission and signal reception (Bruker Biospin). Localizer images were acquired using a spin-echo sequence, and corrections of slice angulation were performed, if necessary. RARE (rapid acquisition with relaxation enhancement) sequences (repetition time $[\mathrm{TR}]=2,500$ $\mathrm{ms}$, echo time $[\mathrm{TE}]=36.7 \mathrm{~ms}$, slice thickness $=1 \mathrm{~mm}$ ) in axial and coronal orientation were used to determine exact positioning of the lower part of the mouse body. Afterwards, a coronal MSME (multi-slicemulti-echo) spin-echo sequence with $\mathrm{TE}=8.6 \mathrm{~ms}$, TR $=453 \mathrm{~ms}$, field of view $(\mathrm{FOV})=7 \times 7 \mathrm{~cm}^{2}$, matrix size $($ MTX $)=512 \times 256$, and a slice thickness of $1 \mathrm{~mm}$ was recorded.

Calorimetry. Measurements of energy expenditure were performed at $23^{\circ} \mathrm{C}$ using an indirect calorimetric system (TSE-Systems). Following 48 hours of acclimatization, $\mathrm{O}_{2}$ consumption and $\mathrm{CO}_{2}$ production were measured every 10 minutes for a total of 48 hours and converted to $\mathrm{kcal} / \mathrm{h}$ using the Weir equation (55).

Total energy expenditure was analyzed using ANCOVA, with body weight as covariate as previously suggested (56). Home-cage locomotor activity was determined using an ActiMot infrared light beam system integrated in the calorimetry system.

Glucose tolerance test. After overnight fasting, glucose was administered i.p. at a dose of $1 \mathrm{mg} / \mathrm{g}$ body weight in $o b / o b$ mice and at $2 \mathrm{mg} / \mathrm{g}$ body weight in chow- or HFD-fed animals. Blood samples were taken from the tail vein before and after glucose administration at indicated time points, and glucose levels were measured using a glucometer (Accu-Chek Aviva, Roche).

Euglycemic-hyperinsulinemic clamp. After fasting overnight, mice were anesthetized with pentobarbital. A catheter was placed into the tail vein, and $50 \mu 1 / \mathrm{h}$ PBS was infused for 30 minutes. A $30-\mu \mathrm{l}$ bolus of insulin solution $(40 \mathrm{IU} / \mathrm{ml}$ ) was given via the catheter, followed by continuous infusion of insulin solution at 50 $\mu \mathrm{l} / \mathrm{h}$. Blood glucose was measured with a glucometer before insulin infusion and 5, 10, 15, 20, 30, 40, 50, $60,70,80$, and 90 minutes after the start of continuous insulin infusion. Glucose infusion (12.5\% glucose in PBS) was started when needed and adjusted to maintain a blood glucose concentration equal to the basal value. The GIR during the last 30 minutes is the steady-state GIR.

Etanercept treatment in vitro and in vivo. Isolated adipocytes from Adipoq $\mathrm{CreER}^{\mathrm{T} 2+/-} ; \mathrm{TAK} 1^{\mathrm{f} / \mathrm{fl}}$ and control animals were incubated in DMEM/FBS/antibiotic solution in the presence of TNF- $\alpha(20 \mathrm{ng} / \mathrm{ml})$ and etanercept $(0.1 \mu \mathrm{g} / \mathrm{ml}$ and $1.0 \mu \mathrm{g} / \mathrm{ml})$ at $37^{\circ} \mathrm{C}$ and $5 \% \mathrm{CO}_{2}$ for 48 hours. The apoptotic rate was measured by determining caspase- 3 activity.

On days 1, 3, and 5 after tamoxifen induction, $100 \mathrm{mg} / \mathrm{kg}$ etanercept was injected i.p. After 48 hours, animals were sacrificed, adipocytes were isolated from the epididymal fat pads, and caspase- 3 activity was measured.

Liver triglyceride content. For creation of saponified and neutralized liver extracts, 100-300 mg of liver tissue was pre-weighed into microfuge tubes, and $350 \mu$ ethanolic $\mathrm{KOH}$ ( 2 parts ethanol:1 part $\mathrm{KOH}$ ) was added and incubated overnight at $55^{\circ} \mathrm{C}$. The volume was brought to $1,000 \mu 1$ by adding $\mathrm{H}_{2} \mathrm{O}$ :ethanol $(1: 1)$, and samples were centrifuged for 5 minutes. The supernatant was transferred to a new tube and the volume was adjusted to $1,200 \mu \mathrm{l}$ by adding $\mathrm{H}_{2} \mathrm{O}$ :ethanol (1:1). Two hundred microliters were transferred to a new tube, $215 \mu \mathrm{l}$ of $1 \mathrm{M} \mathrm{MgCl}_{2}$ was added, and the samples were incubated on ice for 10 minutes. After another centrifugation step the supernatant was transferred to a new tube and triglyceride concentration was determined using the Triglyceride GPO-PAP kit (RANDOX Laboratories Ltd.). Liver triglyceride content was calculated as mg triglycerides per gram liver tissue.

Statistics. Data are displayed as mean \pm SEM. $n$ indicates the number of animals per experimental group or number of independent experiments. Comparisons between groups were performed with a 2 -tailed unpaired $t$ test or ANOVA. A $P$ value less than or equal to 0.05 was considered significant.

Study approval. Animal experiments were approved by the Institutional Animal Care and Use Committee of the Regierungspräsidia Karlsruhe and Darmstadt.

\section{Author contributions}

AS performed most of the experiments, evaluated data, and wrote the manuscript. PS performed expression analysis. CT helped with analysis of metabolic phenotyping. AW performed MRI. NW supervised the project and commented on the manuscript. SO conceived the study, supervised the project, and wrote the manuscript. 


\section{Acknowledgments}

We thank Svea Hümmer for excellent secretarial work, Ulrike Schlapp for technical assistance, and Carola Meyer for help with the evaluation of calorimetry data.

Address correspondence to: Antonia Sassmann-Schweda, Ludwigstrasse 43, 61231 Bad Nauheim, Germany. Phone: 49.6032.705.1206; E-mail: antonia.sassmann@mpi-bn.mpg.de.

1. Haslam DW, James WP. Obesity. Lancet. 2005;366(9492):1197-1209.

2. Gilbert CA, Slingerland JM. Cytokines, obesity, and cancer: new insights on mechanisms linking obesity to cancer risk and progression. Аnпu Rev Med. 2013;64:45-57.

3. Finucane MM, et al. National, regional, and global trends in body-mass index since 1980: systematic analysis of health examination surveys and epidemiological studies with 960 country-years and 9.1 million participants. Lancet. 2011;377(9765):557567.

4. Olefsky JM, Glass CK. Macrophages, inflammation, and insulin resistance. Annu Rev Physiol. 2010;72:219-246.

5. Xu H, et al. Chronic inflammation in fat plays a crucial role in the development of obesity-related insulin resistance. J Clin Invest. 2003;112(12):1821-1830.

6. Weisberg SP, McCann D, Desai M, Rosenbaum M, Leibel RL, Ferrante AW Jr. Obesity is associated with macrophage accumulation in adipose tissue. $J$ Clin Invest. 2003;112(12):1796-1808.

7. Lumeng CN, Saltiel AR. Inflammatory links between obesity and metabolic disease. J Clin Invest. 2011;121(6):2111-2117.

8. Osborn O, Olefsky JM. The cellular and signaling networks linking the immune system and metabolism in disease. Nat Med. 2012;18(3):363-374.

9. Sabio G, et al. A stress signaling pathway in adipose tissue regulates hepatic insulin resistance. Science. 2008;322(5907):15391543.

10. Shi H, Kokoeva MV, Inouye K, Tzameli I, Yin H, Flier JS. TLR4 links innate immunity and fatty acid-induced insulin resistance. J Clin Invest. 2006;116(11):3015-3025.

11. Sun K, Kusminski CM, Scherer PE. Adipose tissue remodeling and obesity. J Clin Invest. 2011;121(6):2094-2101.

12. Gregor MF, Hotamisligil GS. Inflammatory mechanisms in obesity. Annu Rev Immunol. 2011;29:415-445.

13. Chawla A, Nguyen KD, Goh YP. Macrophage-mediated inflammation in metabolic disease. Nat Rev Immunol. 2011;11(11):738749.

14. Cildir G, Akincilar SC, Tergaonkar V. Chronic adipose tissue inflammation: all immune cells on the stage. Trends Mol Med. 2013;19(8):487-500.

15. Salans LB, Cushman SW, Weismann RE. Studies of human adipose tissue. Adipose cell size and number in nonobese and obese patients. J Clin Invest. 1973;52(4):929-941.

16. Spalding KL, et al. Dynamics of fat cell turnover in humans. Nature. 2008;453(7196):783-787.

17. Hirsch J, Batchelor B. Adipose tissue cellularity in human obesity. Clin Endocrinol Metab. 1976;5(2):299-311.

18. Ryden M, et al. Lipolysis--not inflammation, cell death, or lipogenesis--is involved in adipose tissue loss in cancer cachexia. Cancer. 2008;113(7):1695-1704.

19. Lofgren $P$, et al. Long-term prospective and controlled studies demonstrate adipose tissue hypercellularity and relative leptin deficiency in the postobese state. J Clin Endocrinol Metab. 2005;90(11):6207-6213.

20. Sakurai H. Targeting of TAK1 in inflammatory disorders and cancer. Trends Pharmacol Sci. 2012;33(10):522-530.

21. Dai L, Aye Thu C, Liu XY, Xi J, Cheung PC. TAK1, more than just innate immunity. IUBMB Life. 2012;64(10):825-834.

22. Arslan SC, Scheidereit C. The prevalence of TNFalpha-induced necrosis over apoptosis is determined by TAK1-RIP1 interplay. PLoS One. 2011;6(10):e26069.

23. Kajino-Sakamoto R, et al. Enterocyte-derived TAK1 signaling prevents epithelium apoptosis and the development of ileitis and colitis. J Immunol. 2008;181(2):1143-1152.

24. Tang M, et al. TAK1 is required for the survival of hematopoietic cells and hepatocytes in mice. J Exp Med. 2008;205(7):1611-1619.

25. Omori E, et al. TAK1 is a master regulator of epidermal homeostasis involving skin inflammation and apoptosis. J Biol Chem. 2006;281(28):19610-19617.

26. Inokuchi S, et al. Disruption of TAK1 in hepatocytes causes hepatic injury, inflammation, fibrosis, and carcinogenesis. Proc Natl Acad Sci U S A. 2010;107(2):844-849.

27. Singh A, et al. TAK1 inhibition promotes apoptosis in KRAS-dependent colon cancers. Cell. 2012;148(4):639-650.

28. Li L, Chen Y, Doan J, Murray J, Molkentin JD, Liu Q. A TAK1 signaling pathway critically regulates myocardial survival and remodeling. Circulation. 2014; 130(24):2162-2172.

29. Sassmann A, Offermanns S, Wettschureck N. Tamoxifen-inducible Cre-mediated recombination in adipocytes. Genesis. 2010;48(10):618-625.

30. Sato S, et al. Essential function for the kinase TAK1 in innate and adaptive immune responses. Nat Immunol. 2005;6(11):10871095 .

31. Shim JH, et al. TAK1, but not TAB1 or TAB2, plays an essential role in multiple signaling pathways in vivo. Genes Dev. 2005;19(22):2668-2681.

32. Cinti S, et al. Adipocyte death defines macrophage localization and function in adipose tissue of obese mice and humans. $J$ Lipid Res. 2005;46(11):2347-2355.

33. Salans LB, Horton ES, Sims EA. Experimental obesity in man: cellular character of the adipose tissue. J Clin Invest. 1971;50(5):1005-1011.

34. Alkhouri N, et al. Adipocyte apoptosis, a link between obesity, insulin resistance, and hepatic steatosis. J Biol Chem. 2010;285(5):3428-3438. 
35. Fischer-Posovszky P, Wang QA, Asterholm IW, Rutkowski JM, Scherer PE. Targeted deletion of adipocytes by apoptosis leads to adipose tissue recruitment of alternatively activated M2 macrophages. Endocrinology. 2011;152(8):3074-3081.

36. Lumeng CN, Bodzin JL, Saltiel AR. Obesity induces a phenotypic switch in adipose tissue macrophage polarization. JClin Invest. 2007;117(1):175-184.

37. Lumeng CN, DelProposto JB, Westcott DJ, Saltiel AR. Phenotypic switching of adipose tissue macrophages with obesity is generated by spatiotemporal differences in macrophage subtypes. Diabetes. 2008;57(12):3239-3246.

38. Bettermann K, et al. TAK1 suppresses a NEMO-dependent but NF-kappaB-independent pathway to liver cancer. Cancer Cell. 2010;17(5):481-496.

39. Thiefes A, et al. Simultaneous blockade of NFkappaB, JNK, and p38 MAPK by a kinase-inactive mutant of the protein kinase TAK1 sensitizes cells to apoptosis and affects a distinct spectrum of tumor necrosis factor [corrected] target genes. $J$ Biol Chem. 2005;280(30):27728-27741.

40. Wu J, Cohen P, Spiegelman BM. Adaptive thermogenesis in adipocytes: is beige the new brown? Genes Dev. 2013;27(3):234-250.

41. Bonet ML, Oliver P, Palou A. Pharmacological and nutritional agents promoting browning of white adipose tissue. Biochim Biophys Acta. 2013;1831(5):969-985.

42. Harms M, Seale P. Brown and beige fat: development, function and therapeutic potential. Nat Med. 2013;19(10):1252-1263.

43. Chiang SH, et al. The protein kinase IKKepsilon regulates energy balance in obese mice. Cell. 2009;138(5):961-975.

44. Huang Z, Chen D, Zhang K, Yu B, Chen X, Meng J. Regulation of myostatin signaling by c-Jun N-terminal kinase in C2C12 cells. Cell Signal. 2007;19(11):2286-2295.

45. Philip B, Lu Z, Gao Y. Regulation of GDF-8 signaling by the p38 MAPK. Cell Signal. 2005;17(3):365-375.

46. Koncarevic A, et al. A novel therapeutic approach to treating obesity through modulation of TGFbeta signaling. Endocrinology. 2012;153(7):3133-3146.

47. Fournier B, et al. Blockade of the activin receptor IIb activates functional brown adipogenesis and thermogenesis by inducing mitochondrial oxidative metabolism. Mol Cell Biol. 2012;32(14):2871-2879.

48. Hui XY, et al. Adiponectin enhances cold-induced browning of subcutaneous adipose tissue via promoting M2 macrophage proliferation. Cell Metabolism. 2015;22(2):279-290.

49. Qiu Y, et al. Eosinophils and type 2 cytokine signaling in macrophages orchestrate development of functional beige fat. Cell. 2014;157(6):1292-1308.

50. Nguyen $\mathrm{KD}$, et al. Alternatively activated macrophages produce catecholamines to sustain adaptive thermogenesis. Nature 2011;480(7375):104-108.

51. Lee MW, et al. Activated type 2 innate lymphoid cells regulate beige fat biogenesis. Cell. 2015;160(1-2):74-787.

52. Rodbell M. Metabolism of isolated fat cells. I. Effects of hormones on glucose metabolism and lipolysis. J Biol Chem. 1964;239:375-380.

53. Chen HC, Farese RV Jr. Determination of adipocyte size by computer image analysis. J Lipid Res. 2002;43(6):986-989

54. Bourgeois F, Alexiu A, Lemonnier D. Dietary-induced obesity: effect of dietary fats on adipose tissue cellularity in mice. Br J Nutr. 1983;49(1):17-26.

55. Weir JB. New methods for calculating metabolic rate with special reference to protein metabolism. J Physiol. 1949;109(1-2):1-9. 56. Tschop MH, et al. A guide to analysis of mouse energy metabolism. Nat Methods. 2012;9(1):57-63. 